\title{
Short-range correlations and the nuclear EMC effect in deuterium and helium-3
}

\author{
E. P. Segarra $\odot,{ }^{1}$ J. R. Pybus, ${ }^{1}$ F. Hauenstein $\odot,{ }^{1,2}$ D. W. Higinbotham $\odot,{ }^{3}$ G. A. Miller $\odot,{ }^{4}$ E. Piasetzky, ${ }^{5}$ A. Schmidt $\odot,{ }^{6}$ \\ M. Strikman, ${ }^{7}$ L. B. Weinstein $\odot,{ }^{2}$ and O. Hen $\odot^{1, *}$ \\ ${ }^{1}$ Department of Physics, Massachusetts Institute of Technology, Cambridge, Massachusetts 02139, USA \\ ${ }^{2}$ Department of Physics, Old Dominion University, Norfolk, Virginia 23529, USA \\ ${ }^{3}$ Thomas Jefferson National Accelerator Facility, Newport News, Virginia 23606, USA \\ ${ }^{4}$ Department of Physics, University of Washington, Seattle, Washington 98195-1560, USA \\ ${ }^{5}$ School of Physics and Astronomy, Tel Aviv University, Tel Aviv 69978, Israel \\ ${ }^{6}$ Department of Physics, George Washington University, Washington, DC 20052, USA \\ ${ }^{7}$ Department of Physics, Pennsylvania State University, University Park, Pennsylvania 16802, USA
}

(Received 21 January 2021; revised 30 March 2021; accepted 5 May 2021; published 22 June 2021)

\begin{abstract}
The EMC effect in deuterium and helium-3 is studied using a convolution formalism that allows isolating the impact of high-momentum nucleons in short-ranged correlated (SRC) pairs. We assume that the modification of the structure function of bound nucleons is given by a universal (i.e., nucleus independent) function of their virtuality, and find that the effect of such modifications is dominated by nucleons in SRC pairs. This SRC-dominance of nucleon modifications is observed despite the fact that the bulk of the nuclear inelastic scattering cross-section comes from interacting with low-momentum nucleons. These conclusions are found to be robust to model details including nucleon modification function parametrization, free nucleon structure function, and treatment of nucleon motion effects. While existing data cannot discriminate between such model details, we present predictions for measured, but not yet published, tritium EMC effect and tagged nucleon structure functions in deuterium that are sensitive to the neutron structure functions and bound nucleon modification functions.
\end{abstract}

DOI: 10.1103/PhysRevResearch.3.023240

\section{INTRODUCTION}

Determining the underlying cause of the modification of the partonic structure of nucleons bound in atomic nuclei, known as the EMC effect [1-7], is an outstanding question in nuclear physics. Decades after its discovery, there is still no universally accepted explanation for the origin of the EMC effect [8-11], despite a large number of high-precision measurements in a wide variety of atomic nuclei.

Modern models of the EMC effect account for both "conventional" nuclear physics effects such as Fermi-motion and binding, as well as for the more "exotic" effects of nucleon modification [10-14], especially considering nucleon off-shellness $[15,16]$. The conventional nuclear physics effects are well understood and cannot reproduce experimental data alone, especially when including Drell-Yan data [11,17]. While required to reproduce experimental data, nucleon modification models are far less constrained and their microscopic origin is debated [11].

\footnotetext{
*Corresponding author: hen@mit.edu

Published by the American Physical Society under the terms of the Creative Commons Attribution 4.0 International license. Further distribution of this work must maintain attribution to the author(s) and the published article's title, journal citation, and DOI.
}

An observed correlation between the magnitude of the EMC effect and the relative abundance of short-range correlated (SRC) nucleon pairs in different nuclei [7,18-20] suggests that the EMC effect is driven by the modification of nucleons in SRC pairs. SRCs are pairs of strongly interacting nucleons at short distances. Nucleons in SRC pairs have large spatial overlap between their quark distributions and are highly off-shell $\left(E^{2} \neq|\mathbf{p}|^{2}+m^{2}\right)$, which makes them prime candidates for structure modification.

Most recently, it has been demonstrated [7,21] that the EMC effect in nuclei from helium-3 $\left({ }^{3} \mathrm{He}\right)$ to lead can be explained by a single effective universal modification function (UMF) of nucleons in SRC pairs. The UMF was constructed to be as model independent as possible. It is insensitive to the largely-unknown free-neutron structure function, $F_{2}^{n}$, and accounts for both conventional nuclear effects, such as the scheme dependence of the deuteron wave function, and nucleon motion effects, as well as more exotic nucleon modification effects.

Here we study the EMC effect using a convolution formalism that allows us to separate the mean field and short range correlation contributions of nucleon modification effects to the total UMF. We consider only light nuclei (the deuteron and ${ }^{3} \mathrm{He}$ ), for which exact nuclear wave functions are available, and nucleon modification effects can be isolated. The sensitivity of the convolution formalism to parametrization of the nucleon modification function $F_{2}^{n}$ and the treatment of nucleon motion effects is studied. 
The convolution work described here is similar to the works of [22,23], although here we only focus on deuterium and helium-3 to make use of the exactly calculable nuclear dynamics that is available only for these systems. We further study a wide model phase space by considering different models of the free neutron structure function, nuclear motion dynamics, and bound nucleon modification functions.

We find that, as expected, the bulk of the structure-function comes from interactions with low-momentum nucleons. However, nucleon modification effects, which are required for a complete reproduction of the measured data, are dominated by nucleons in SRC pairs. We also find that existing data cannot discriminate between different $F_{2}^{n}$ models or different parametrizations of bound nucleon modification functions. We predict new observables that can constrain these model inputs, including the tritium EMC effect, sensitive to $F_{2}^{n}$, and deuterium tagged nucleon structure functions, sensitive to bound nucleon modification functions. These predictions will soon be tested by data from the MARATHON [24], BAND [25], and LAD [26] Collaborations.

\section{FORMALISM}

\section{A. $F_{2}^{A}$ convolution approximation}

In order to study the EMC effect in a framework that allows us to understand its dependence on nucleon momentum and off-shellness, we calculate the nuclear structure function $F_{2}^{A}\left(x_{B}\right)$, using the nuclear convolution model for leptonnucleus DIS [8,27-30]:

$$
\begin{aligned}
& F_{2}^{A}\left(x_{B}\right)= \\
& \begin{aligned}
\frac{1}{A} \int_{x_{B}}^{A} \frac{d \alpha}{\alpha} \int_{-\infty}^{0} d v\left[Z \tilde{\rho}_{p}^{A}(\alpha, v) F_{2}^{p}(\tilde{x})+N \tilde{\rho}_{n}^{A}(\alpha, v) F_{2}^{n}(\tilde{x})\right] \\
\quad \times\left(1+v f^{\mathrm{off}}(\tilde{x})\right) \\
=\frac{1}{A} \int_{x_{B}}^{A} \frac{d \alpha}{\alpha} \int_{-\infty}^{0} d v F_{2}^{p}(\tilde{x})\left[Z \tilde{\rho}_{p}^{A}(\alpha, v)+N \tilde{\rho}_{n}^{A}(\alpha, v) \frac{F_{2}^{n}(\tilde{x})}{F_{2}^{p}(\tilde{x})}\right] \\
\quad \times\left(1+v f^{\mathrm{off}(\tilde{x})),}\right.
\end{aligned}
\end{aligned}
$$

where $x_{B}=Q^{2} /\left(2 m_{N} v\right), Q^{2}$ is the four-momentum transfer squared, $m_{N}$ is the nucleon mass, and $v$ is the energy transfer (Fig. 1). $\tilde{x}=\frac{Q^{2}}{2 p \cdot q}$ where $q$ is the four-momentum of the virtual photon and $p$ is the initial four-momentum of the struck off-shell nucleon. $\tilde{x}$ reduces to $\frac{x_{B}}{\alpha} \frac{m_{N} A}{m_{A}}$ in the Bjorken limit with lightcone momentum fraction $\alpha=A\left(E+p_{z}\right) / m_{A}$ [see Appendices for finite energy corrections to Eq. (A1) at low $Q^{2}$ ]. Here $z$ is opposite to the direction of the virtual photon, and $v=\left(E^{2}-|\mathbf{p}|^{2}-m_{N}^{2}\right) / m_{N}^{2}$ is the bound nucleon fractional virtuality. The functions $\tilde{\rho}_{N}^{A}(\alpha, v)$ are the nucleon $(N=p$ or $n)$ lightcone momentum and virtuality distributions in nucleus $A$, defined below. $F_{2}^{p}(\tilde{x})$ and $F_{2}^{n}(\tilde{x})$ are the free proton and neutron structure functions. We note that Eq. (A1) arises from a four-dimensional integral involving $d^{4} p$, where we perform a change of variables to $\alpha, \nu$, and integrate over the remaining degrees of freedom [8]. For brevity we omit their explicit $Q^{2}$ dependencies but note that $F_{2}^{p}, F_{2}^{n}$, and $F_{2}^{A}$
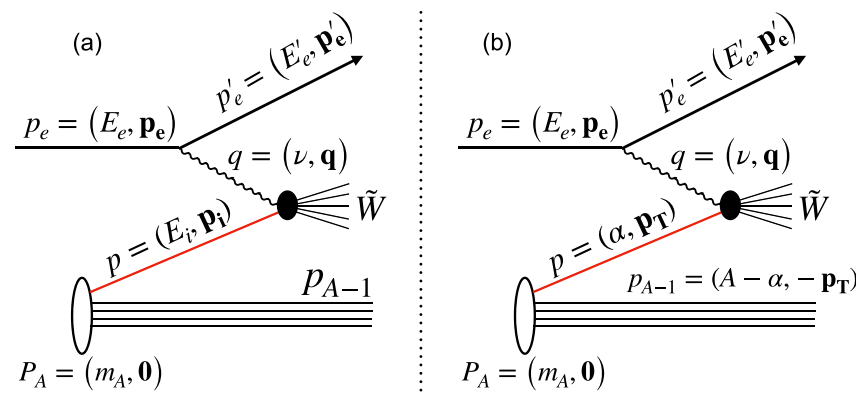

FIG. 1. Reaction diagram for lepton-nuclear deep inelastic scattering in a factorized plane wave impulse approximation for (a) spectral function kinematics and (b) lightcone kinematics. Red lines represent off-shell nucleons. See text for details.

are always evaluated at the same $Q^{2}$ value. $f^{\text {off }}(\tilde{x})$ is a universal off-shell nucleon modification function, assumed here to be the same for neutrons and protons and for all nuclei. In Eq. (A1), we take the off-shell effect to be linear in $v$ (i.e., $\left.1+v f^{\text {off }}(\tilde{x})\right)$ as a first-order Taylor expansion in virtuality; see Ref. [31] for additional discussion.

\section{B. Lightcone densities}

In our convolution, traditional nuclear contributions to the EMC effect such as nucleon motion and binding are treated within the one-body lightcone momentum and virtuality distribution $\tilde{\rho}_{N}^{A}(\alpha, v)$. It describes the joint probability to find a nucleon $(n$ or $p)$ in a nucleus $A$ with lightcone momentum fraction $\alpha$ and fractional virtuality $v$. Integrating over fractional virtuality defines the lightcone momentum distribution of a nucleon

$$
\rho_{N}^{A}(\alpha)=\int_{-\infty}^{0} d v \tilde{\rho}_{N}^{A}(\alpha, v),
$$

which is normalized herein according to the baryon sum rule:

$$
\int_{0}^{A} \frac{d \alpha}{\alpha} \rho_{N}^{A}(\alpha) \equiv 1 .
$$

To avoid producing an artificial EMC-like effect in nucleon-only models when used in Eq. (A1) [28], $\rho_{N}^{A}(\alpha)$ must also satisfy the momentum sum rule:

$$
\frac{1}{A} \int_{0}^{A} \frac{d \alpha}{\alpha} \alpha\left(Z \rho_{p}^{A}(\alpha)+N \rho_{n}^{A}(\alpha)\right)=1 .
$$

It is necessary to know the functional form of $\tilde{\rho}_{N}^{A}(\alpha, v)$ to proceed further. Although the nuclear wave functions for nuclei with $A=2$ and $A=3$ have been well computed, they do not suffice to unambiguously yield the lightcone momentum distributions and their dependence on virtuality. Handling this issue on a fundamental level would require a first-principles light-front calculation including the effects of off-mass-shell dependence. Such a calculation could be done by solving the relevant Bethe-Salpeter equation, but does not yet exist.

Therefore, we consider here two approximations to estimate $\tilde{\rho}(\alpha, v)$ : a spectral-function (SF) approximation, where the momentum sum rule is violated if only nucleonic degrees of freedom are taken into account, and a generalized contact formalism lightcone (GCF-LC) approximation. 


\section{Spectral function approximation}

The nuclear spectral function $S(E, p)$ defines the probability for finding a nucleon in the nucleus with momentum $p$ and nucleon energy $E$. Exactly calculable spectral functions are available for light nuclei and allow calculating the nuclear lightcone distributions as [32,33]

$$
\begin{aligned}
\tilde{\rho}_{N, S F}^{A}(\alpha, v)= & \int d E d^{3} \mathbf{p} S_{N}^{A}(E, p) \cdot \frac{E+p_{z}}{E} \\
& \times \delta\left(\alpha-\frac{A p^{+}}{P^{+}}\right) \delta\left(v-\frac{E^{2}-|\mathbf{p}|^{2}-m_{N}^{2}}{m_{N}^{2}}\right),
\end{aligned}
$$

where $p=|\mathbf{p}|, p^{+} \equiv E+p_{z}=m_{A} \alpha / A$ is the plus-component of the momentum of the struck nucleon, $P^{+}=m_{A}$ is the pluscomponent of the momentum of the nucleus $A$, and $m_{A}$ is the nucleus mass.

The flux factor $\left(E+p_{z}\right)$ is introduced to help satisfy the momentum sum rule [28]. The $\frac{1}{E}$ factor ensures SF-based lightcone distribution functions are appropriately normalized according to the Baryon sum rule [Eq. (3)]. However, this also changes the interpretation of $\rho(\alpha)$ from a simple probability density for finding a nucleon in a nucleus with lightcone momentum fraction $\alpha$ (see discussion in Refs. [28,32,33]).

For deuterium, considering a wave function calculated using the AV18 interaction, the momentum sum rule has a negligible violation $(<0.1 \%)$. For ${ }^{3} \mathrm{He}$, using the AV18-based spectral function of Ref. [34], it is violated by $\leqslant 1 \%$. This small violation is expected to produce an artificial EMC effect [28] that should result in a smaller nucleon modification effect required to explain the experimental data.

\section{Generalized contact formalism lightcone approximation}

To fully satisfy the ${ }^{3} \mathrm{He}$ momentum sum rule, we examine an alternative approach for calculating $\tilde{\rho}_{N}^{A}(\alpha, v)$ using a scale-separation approximation where the lightcone density function is separated into a mean-field (single-nucleon) part and an SRC part [20,35-38]:

$$
\tilde{\rho}_{N, G C F-L C}^{A}(\alpha, v)=\tilde{\rho}_{N, G C F, S R C}^{A}(\alpha, v)+\tilde{\rho}_{N, M F}^{A}(\alpha, v) .
$$

The SRC part of the lightcone density can be formulated by integrating over the lightcone SRC decay function $[38,39]$, which describes the distribution of the momentum of the struck nucleon as well as its partner, here denoted the "spectator" nucleon:

$$
\begin{aligned}
\tilde{\rho}_{N, G C F, S R C}^{A}(\alpha, v)= & \int d^{2} \mathbf{p}_{\perp} \frac{d \alpha_{s}}{\alpha_{s}} d^{2} \mathbf{p}_{s}^{\perp} \rho_{S R C}^{N}\left(\alpha, \mathbf{p}^{\perp}, \alpha_{s}, \mathbf{p}_{s}^{\perp}\right) \\
& \times \delta\left(v-\frac{p^{-}\left(m_{A} / A\right) \alpha-\mathbf{p}_{\perp}^{2}-m_{N}^{2}}{m_{N}^{2}}\right),
\end{aligned}
$$

where

$$
\begin{aligned}
p^{-} & =P^{-}-p_{s}^{-}-p_{A-2}^{-} \\
& =m_{A}-\frac{m_{N}^{2}+\left(\mathbf{p}_{s}^{\perp}\right)^{2}}{\left(m_{A} / A\right) \alpha_{s}}-\frac{m_{A-2}^{2}+\left(\mathbf{p}_{C M}^{\perp}\right)^{2}}{\left(m_{A} / A\right)\left(A-\alpha-\alpha_{s}\right)}
\end{aligned}
$$

is the off-mass shell minus-component of the struck nucleon's momentum, $\alpha_{s}$ is the spectator nucleon lightcone fraction, $\mathbf{p}_{\perp}$ and $\mathbf{p}_{s, \perp}$ are the transverse momentum of the struck nucleon and the spectator, respectively, and $\mathbf{p}_{C M}^{\perp}=\mathbf{p}_{\perp}+\mathbf{p}_{s, \perp} \cdot \rho_{S R C}^{N}$ is a two-body (i.e., pair) lightcone density given by a convolution of the pair center-of-mass and relative momentum densities. See Ref. [38] and Appendices for details.

The mean-field part of the lightcone density is taken from the spectral functions using a linearized approximation, similar to Eq. (5), but which manifestly preserves the baryon number and momentum sum rules:

$$
\begin{aligned}
\tilde{\rho}_{N, M F}^{A}(\alpha, v)= & \alpha \int_{0}^{m_{N}} d E \int_{0}^{p_{\text {cutoff }}} d^{3} \mathbf{p} S_{N}^{A}(E, p) \\
& \times \delta\left(\alpha-1-\frac{A p_{z}}{P^{+}}\right) \delta\left(v-\frac{E^{2}-|\mathbf{p}|^{2}-m_{N}^{2}}{m_{N}^{2}}\right) .
\end{aligned}
$$

The cutoff momentum, defined in the laboratory frame, $p_{\text {cutoff }}=240 \mathrm{MeV} / c$ for ${ }^{3} \mathrm{He}$ and was chosen such that the fraction of SRC pairs was equal to that extracted from $a b$ initio many-body calculations $(10.1 \%$ for neutrons and $5.9 \%$ for protons) $[40,41]$.

We note that the exact momentum onset for SRC dominance is not exactly known, it is generally taken to be around the nuclear Fermi momentum. This is supported by both experimental SRC scaling onset observations at $275 \pm$ $25 \mathrm{MeV} / \mathrm{c}$ [42] and calculations of infinite nuclear matter [43-45]. While light nuclei, such as helium-3, do not have a well defined Fermi momentum, our choice of a value slightly lower than the Fermi-momentum of medium nuclei is reasonable and as explained above also consistent with SRC probabilities (contact terms) extracted from ab-initio calculations $[40,41]$.

We emphasize that the momentum sum rule for $\tilde{\rho}_{N, G C F-L C}^{A}(\alpha, v)$ is manifestly satisfied in this approximation and that the resulting GCF-LC density has a mean value of unity, in contrast to that obtained in the SF approximation (Fig. 2). We note that this is possible because the implementation of SRC center-of-mass motion in the LC decay function is performed on the lightfront (see Ref. [38] for full details).

\section{STRUCTURE, FUNCTION, AND MODIFICATION MODELS}

We compute Eq. (A1) using parametrizations of $f^{\text {off }}(\tilde{x})$, $F_{2}^{p}(\tilde{x})$, and $\frac{F_{2}^{n}(\tilde{x})}{F_{2}^{p}(\tilde{x})}$, and both $\tilde{\rho}_{N, S F}^{A}$ and $\tilde{\rho}_{N, G C F-L C}^{A}$. For the modification function $f^{\text {off }}(\tilde{x})$ we consider three models:

$$
\begin{gathered}
f_{\text {const }}^{\text {off }}(\tilde{x})=C, \\
f_{\operatorname{lin} x}^{\text {off }}(\tilde{x})=a+b \cdot \tilde{x} \\
f_{K P, C J}^{\text {off }}(\tilde{x})=C\left(x_{0}-\tilde{x}\right)\left(x_{1}-\tilde{x}\right)\left(1+x_{0}-\tilde{x}\right)
\end{gathered}
$$

where $f_{\text {const }}^{\text {off }}$ assumes a virtuality-dependent modification model that is independent of $\tilde{x}$, and $f_{\operatorname{lin} x}^{\text {off } x}$ is also linearly dependent on $\tilde{x}$. The free parameters of these parametrizations $(C$, $a$, and $b$ ) are determined by fitting Eq. (A1) to experimental data as detailed below. 

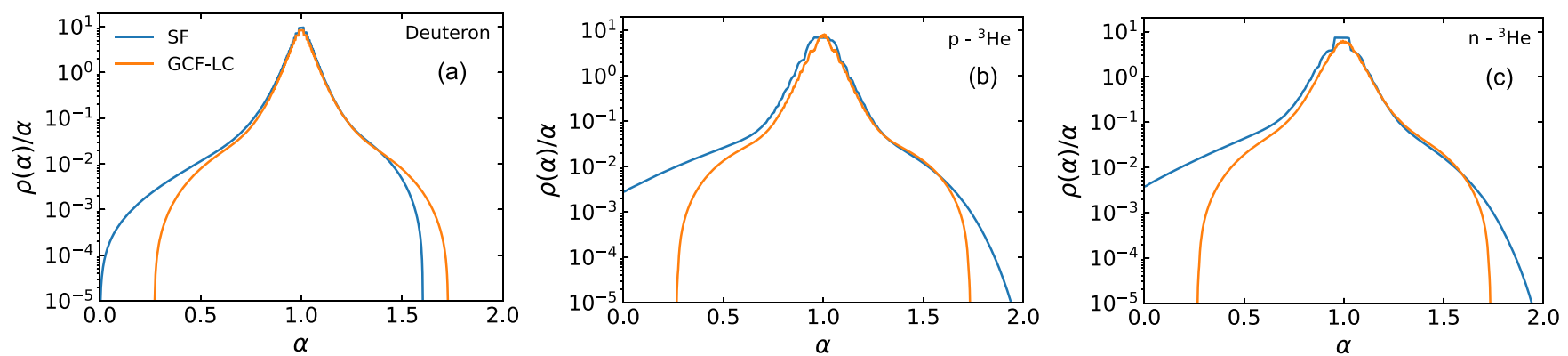

FIG. 2. Lightcone momentum distributions $\rho(\alpha)$ for deuteron (a), protons (b), and neutrons (c) in ${ }^{3} \mathrm{He}$ calculated using the spectral function (SF) and generalized contact formalism lightcone (GCF-LC) approximations. The discretization visible in the SF distributions (blue lines) is due to the discretization of the spectral function $S(E, p)$ and integration of Eq. (5).

We also use modification functions determined by $\mathrm{KP}$ $\left(f_{K P}^{\text {off }}\right)$ [22] and CJ ( $\left.f_{C J}^{\text {off }}\right)$ [46], who both chose to use a third order polynomial in $\tilde{x}$, albeit with different parameters. These are used here with their original parameters, extracted in Refs. [22,46].

$\frac{F_{2}^{n}(\tilde{x})}{F_{2}^{p}(\tilde{x})}$ was parametrized as

$$
\frac{F_{2}^{n}(\tilde{x})}{F_{2}^{p}(\tilde{x})} \equiv R_{n p}(\tilde{x})=a_{n p}(1-\tilde{x})^{b_{n p}}+c_{n p},
$$

where $R_{n p}(\tilde{x} \rightarrow 1)=c_{n p}$. We fix the $a_{n p}, b_{n p}$, and $c_{n p}$ parameters by fitting Eq. (13) to one of two recent predictions by Segarra et al. [21] and by Arrington et al. [47], which represent two extreme models that capture the spread of current models [21] (see Fig. 3). We further assume that $R_{n p}(\tilde{x})$ has negligible $Q^{2}$ dependence. We note that the original $f_{K P}^{\text {off }}$ and $f_{C J}^{\text {off }}$ extractions were done using $F_{2}^{n} / F_{2}^{p}$ that are respectively similar to the Segarra and Arrington models used herein.

$F_{2}^{p}\left(x_{B}, Q^{2}\right)$ was taken from GD11-P [48]. As DIS data are typically given in the form of $F_{2}^{A} / F_{2}^{d}$ ratios to minimize higher twist effects, the only explicit $Q^{2}$ dependence we assume is that of $F_{2}^{p}\left(x_{B}, Q^{2}\right)$, that is assumed to be negligible in the ratio $F_{2}^{n} / F_{2}^{p}$.

We estimated the parameters of $f_{\text {const }}^{\text {off }}$ and $f_{\text {lin } x}^{\text {off }}$ using a $\chi^{2}$-minimization inference from a simultaneous fit to both

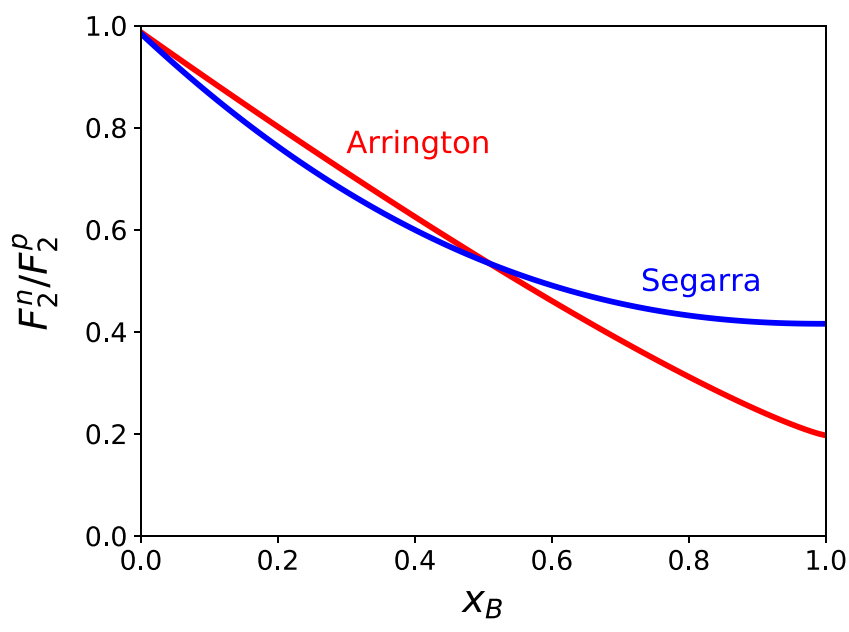

FIG. 3. $F_{2}^{n} / F_{2}^{p}$ parametrizations used in this work that span the current range of models [21]. See text for details.
$F_{2}^{3} \mathrm{He} / F_{2}^{d}$ [6] and $F_{2}^{d} /\left(F_{2}^{p}+F_{2}^{n}\right)$ [49] data for $0.17 \leqslant x_{B} \leqslant$ 0.825 . While data for $F_{2}^{3} \mathrm{He} / F_{2}{ }^{d}$ of [6] extends up to $x_{B} \sim$ 0.9 , these high- $x_{B}$ data are at low-invariant mass $W$. Requiring $W>1.4 \mathrm{GeV}\left(W^{2}>2 \mathrm{GeV}^{2}\right)$ in the fitting procedure limited the data to $x_{B} \leqslant 0.825$. However, we extrapolate our predictions up to $x_{B} \sim 0.95$ for use by future measurements, such as MARATHON [24]. We neglect an explicit quasielastic contribution to our calculation when comparing to data, but this is a minimal contribution when requiring $W>1.4 \mathrm{GeV}$ and restricting our analysis to $x_{B} \leqslant 0.825$. Resonance contributions to $F_{2}^{p}$ and $F_{2}^{n}$ were studied utilizing the Bosted-Christy parametrizations described in Ref. [50], see Appendices. Isoscalar corrections previously applied to $F_{2}^{3} \mathrm{He} / F_{2}^{d}$ data were removed and the quoted experimental normalization uncertainties of each data set were accounted for in the fit. In the calculation of each data point, $F_{2}^{p}$ is evaluated at the $Q^{2}$ value of the data. We performed 16 inference trials for different model assumptions for $\tilde{\rho}(\alpha, v), F_{2}^{n} / F_{2}^{p}$, and $f^{\text {off }}$ (see Table I). For each trial, after minimization, $1 \sigma$ confidence bands were produced from the parameter covariance matrix. When using $f_{K P}^{\text {off }}$ and $f_{C J}^{\text {off }}$, only the error due to renormalization was considered as their off-shell parameters are fixed in our trials.

\section{RESULTS}

\section{A. Inclusive data description}

Figure 4 shows the resulting fit compared to the experimental data using SF densities (GCF-LC densities are shown in Appendices). We performed eight individual fits, switching between the two $F_{2}^{n} / F_{2}^{p}$ models, constant-in- $\tilde{x}$ or linear-in- $\tilde{x}$ off-shell parametrizations, and using either SF or GCF-LC densities. We also show calculations using $f_{K P}^{\text {off }}$ and $f_{C J}^{\text {off }}$. For completeness, Fig. 5 shows the inferred off-shell functions for $f_{\text {const }}^{\text {off }}$ and $f_{\operatorname{lin} x}^{\text {off }}$ for the different convolution frameworks, along with $f_{K P}^{\text {off }}$ [22] and $f_{C J}^{\text {off }}$ [46]. While $f_{K P}^{\text {off }}$ and $f_{C J}^{\text {off }}$ demand zero-crossings in the off-shell function to preserve baryon charge and momentum sum rules, we do not consider this constraint as we restrict our study for $x>0.17$.

From the $\chi^{2}$ values in Table I and the similarity of the different models in Fig. 4, it is clear that the present ${ }^{3} \mathrm{He}$ and ${ }^{2} \mathrm{H}$ data cannot definitively discriminate between the different nucleon motion models (i.e., SF vs GCF-LC) or different neutron structure functions. The data can be adequately 


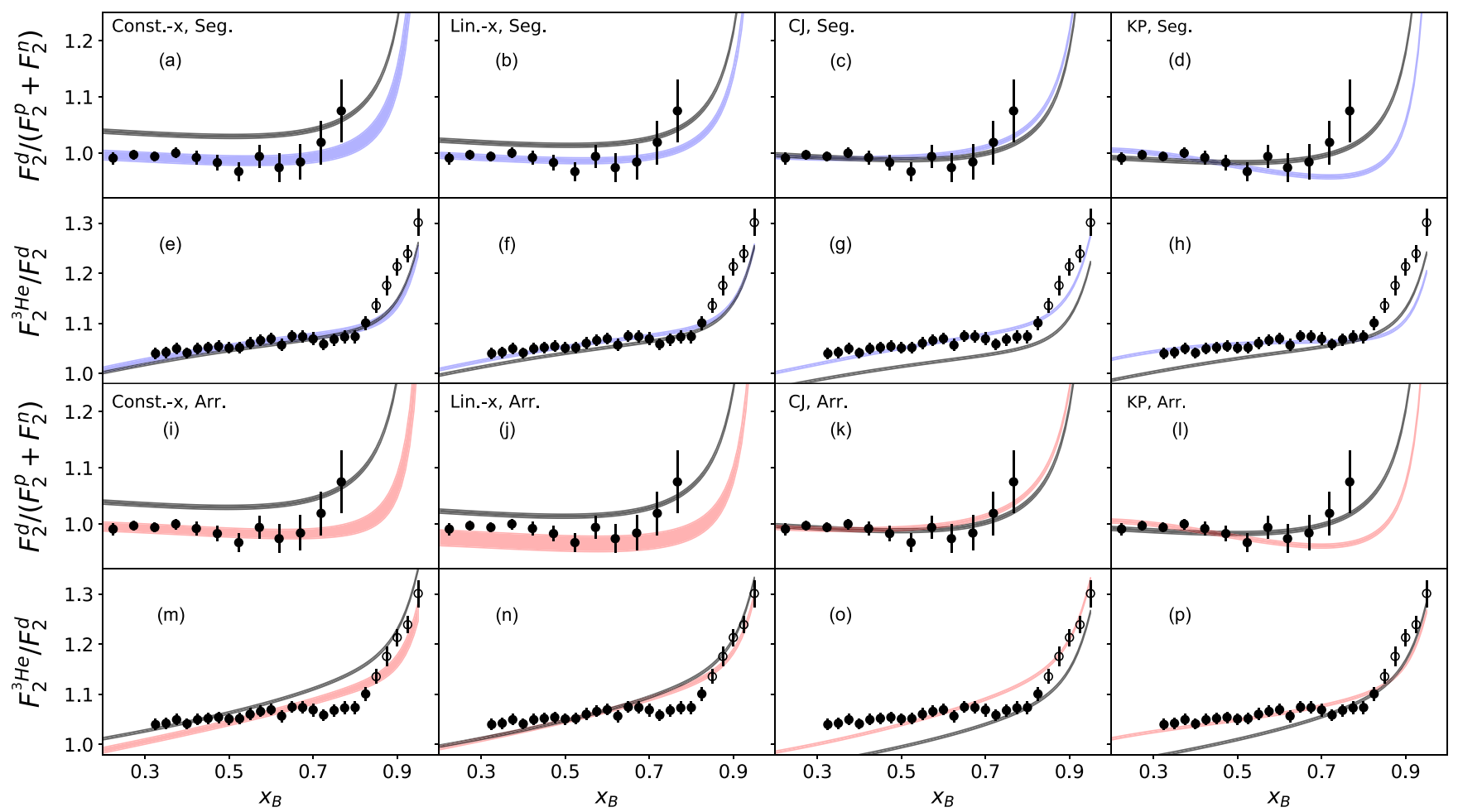

FIG. 4. Convolution results after $\chi^{2}$-minimization procedure using the SF approximation. $1 \sigma$ confidence bands are displayed for each trial, and each band is shown with the renormalization inferred for ${ }^{2} \mathrm{H}$ and ${ }^{3} \mathrm{He}$. The result of no off-shell modification (grey bands) are also shown with the renormalization preferred for that given fit. The data used in the minimization $\left({ }^{2} \mathrm{H}\right.$ data from [49] and ${ }^{3} \mathrm{He}$ data of [6]) are shown as filled circles. Open circles denote data at $W<1.4 \mathrm{GeV}$, which were not used in the fit. [Top row, (a)-(d)] Results for $F_{2}^{d} /\left(F_{2}^{p}+F_{2}^{n}\right)$ with Segarra et al. $F_{2}^{n} / F_{2}^{p}$. [2nd row, (e)-(h)] Results for $F_{2}^{3} H e / F_{2}^{d}$ with Segarra et al. $F_{2}^{n} / F_{2}^{p}$. [3rd row, (i)-(1)] Results for $F_{2}^{d} /\left(F_{2}^{p}+F_{2}^{n}\right)$ with Arrington $F_{2}^{n} / F_{2}^{p}$. [Bottom row, (m)-(p)] Results for $F_{2}^{3} H e / F_{2}^{d}$ with Arrington $F_{2}^{n} / F_{2}^{p}$. In each row, results are shown for different off-shell functions used, in order from left to right: $f_{\text {const }}^{\text {off }}[(\mathrm{a}),(\mathrm{e}),(\mathrm{i}),(\mathrm{m})], f_{\operatorname{lin} x}^{\text {off }}[(\mathrm{b}),(\mathrm{f}),(\mathrm{j}),(\mathrm{n})], f_{C J}^{\text {off }}[(\mathrm{c}),(\mathrm{g}),(\mathrm{k}),(\mathrm{o})], f_{K P}^{\text {off }}[(\mathrm{d}),(\mathrm{h}),(\mathrm{l}),(\mathrm{p})]$. All curves are calculated and extrapolated with the same $Q^{2}$ as the data for ${ }^{2} \mathrm{H}$ and ${ }^{3} \mathrm{He}$.

reproduced even with very different off-shell models. While accounting for nucleon modification improve the reproduction

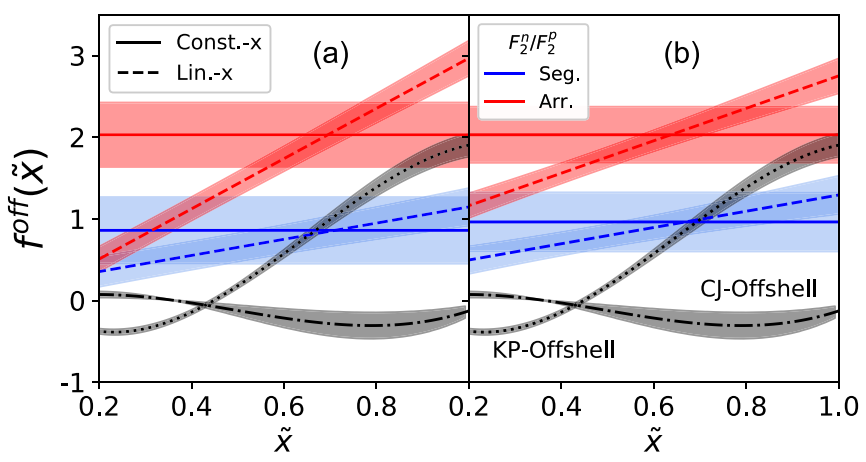

FIG. 5. Off-shell functions $f^{\text {off }}(\tilde{x})$ resulting from $\chi^{2}$-minimization procedure with SF (a) and LC (b) approximations. The blue and red curves were minimization trials using a $F_{2}^{n} / F_{2}^{p}$ fit to two recent predictions by Segarra et al. [21] (Seg.) and Arrington et al. [47] (Arr.), respectively. The two black lines are the off-shell functions as described in $[22,46]$ and were taken as fixed for the minimization procedure, which is why they are identical for both convolution frameworks. $1 \sigma$ confidence bands are displayed for each trial. of the data, in certain cases it can also be reproduced by a "no off-shell modification" calculation, i.e., with $f^{\text {off }}=0$ (see Table I).

Table I and Fig. 4 show a significant systematic improvement for all minimizations when using the Segarra et al. $F_{2}^{n} / F_{2}^{p}$ parametrization (blue curves), particularly for ${ }^{3} \mathrm{He}$. Using $f_{K P}^{\text {off }}$, the calculation does not describe the high- $x_{B}{ }^{2} \mathrm{H}$ data. This is not unexpected as their off-shell function was not fit to BONUS data nor to high- $x_{B}$ deuterium data $(\geqslant 0.8)$ [22]. $f_{K P}^{\text {off }}$ does describe the ${ }^{3} \mathrm{He}$ EMC data markedly well due to the global nature of their analysis, which captures the general EMC trend in a wide range of nuclei. Similarly, when using $f_{C J}^{\text {off }}$, the calculation struggles as much as other models to accurately predict the ${ }^{3} \mathrm{He}$ EMC ratio. However, we note that their global fit does not consider $A>2$ nuclear DIS data. Again, the agreement improves with the use of the Segarra et al. $F_{2}^{n} / F_{2}^{p}$. The resulting off-shell functions from fits with the Segarra et al. $F_{2}^{n} / F_{2}^{p}$ (blue curves) and Arrington (red curves) are independent of the nucleon motion model (SF or GCF-LC), see Fig. 5.

The GCF-LC framework does just as well at describing the ${ }^{3} \mathrm{He}$ data and deuterium data (see Appendices), again, with improvement with the use of the Segarra et al. $F_{2}^{n} / F_{2}^{p}$. In the GCF-LC framework, the high- $x_{B}{ }^{3} \mathrm{He}$ data not used in 
TABLE I. Reduced $\chi^{2}$ results of 20 trials with various model assumptions. $\tilde{\rho}(\alpha, v)$ refers to the approximation that was used for the lightcone virtuality and momentum distribution to calculate Eq. (A1). Similarly, $f^{\text {off }}$ refers to the off-shell functional form used, including a no-modification assumption ( $\left.f^{\text {off }}=0\right)$. There is a systematic increase in $\chi^{2}$ when using $F_{2}^{n} / F_{2}^{p}$ of Arrington [47]. There is also an increase when using GCF-LC approximation to $\tilde{\rho}(\alpha, v)$. We note that when using the KP and CJ off-shell parametrization there are no free parameters fit to data and the quoted $\chi^{2}$ values show the quality of their description of the data without any minimization procedure.

\begin{tabular}{|c|c|c|c|c|c|}
\hline$\tilde{\rho}(\alpha, \nu)$ & $F_{2}^{n} / F_{2}^{p}(\tilde{x})$ & $f^{\text {off }}(\tilde{x})$ & $\chi_{d}^{2}$ & $\chi_{3 \mathrm{He}}^{2}$ & $\chi_{\text {tot }}^{2} /$ d.o.f. \\
\hline \multirow[t]{10}{*}{ SF } & \multirow[t]{5}{*}{ Seg. } & Const.-X & 7.4 & 12.4 & $19.8 / 31=0.63$ \\
\hline & & Lin.-X & 7.7 & 7.7 & $15.4 / 30=0.51$ \\
\hline & & $\mathrm{KP}$ & 12.9 & 12.1 & $25 / 32=0.78$ \\
\hline & & CJ & 6.6 & 23.4 & $30 / 32=0.94$ \\
\hline & & No Mod. & 5.3 & 18.2 & $23.5 / 32=0.73$ \\
\hline & \multirow[t]{5}{*}{ Arr. } & Const.-X & 17.4 & 69.1 & $86.5 / 31=2.79$ \\
\hline & & Lin.-X & 25.9 & 16.0 & $41.9 / 30=1.40$ \\
\hline & & KP & 12.1 & 21.4 & $33.5 / 32=1.05$ \\
\hline & & $\mathrm{CJ}$ & 6.7 & 111.9 & $118.6 / 32=3.71$ \\
\hline & & No Mod. & 5.2 & 98.2 & $103.4 / 32=3.23$ \\
\hline \multirow[t]{10}{*}{ GCF-LC } & \multirow[t]{5}{*}{ Seg. } & Const.-X & 8.4 & 19.2 & $27.6 / 31=0.89$ \\
\hline & & Lin.-X & 7.2 & 16.4 & $23.6 / 30=0.79$ \\
\hline & & KP & 9.8 & 10.5 & $20.3 / 32=0.63$ \\
\hline & & CJ & 11.8 & 26.8 & $38.6 / 32=1.21$ \\
\hline & & No Mod. & 6.3 & 25.0 & $31.3 / 32=0.98$ \\
\hline & \multirow[t]{5}{*}{ Arr. } & Const.-X & 22.9 & 69.3 & $92.2 / 31=2.97$ \\
\hline & & Lin.-x & 25.4 & 53.1 & $78.5 / 30=2.62$ \\
\hline & & KP & 8.7 & 64.5 & $73.2 / 32=2.29$ \\
\hline & & CJ & 12.9 & 110.8 & $123.7 / 32=3.87$ \\
\hline & & No Mod. & 6.2 & 106.2 & $112.4 / 32=3.51$ \\
\hline
\end{tabular}

the fitting procedure (due to having low $W$ ) is not as well described as in the SF framework. We also note that the $f_{C J}^{\text {off }}$ did not use ${ }^{3} \mathrm{He}$ data as a constraint, and, therefore, struggles at describing the data, especially at high $x_{B}$.

\section{B. SRC contribution to nucleon modification}

In addition to being small in magnitude, the resulting off-shell function is dependent on the exact form of $F_{2}^{n} / F_{2}^{p}$. However, the contributions of mean-field nucleons and SRC nucleons to the structure function and off-shell modification are model independent.

Using the inferred parameters from the global fit as described above, we can now separate the contributions of the mean-field and SRC nucleons to the EMC effect. To this end we constructed $F_{2}^{A}=F_{2}^{A}(M F)+F_{2}^{A}(S R C)$ by splitting the integral in Eq. (A1) to contributions of Mean-Field and SRC nucleons. This separation is natural for the GCF-LC approach. For the SF based approach this is done by assigning all nucleons with momenta above a cutoff of $240 \mathrm{MeV} / \mathrm{c}$ as members of SRC pairs. Our findings are largely insensitive to the exact cutoff momentum we choose.

Figure 6 shows the ratio of the structure functions: $\left[F_{2}^{3} \mathrm{He}(S R C)\right] /\left[F_{2}^{3} \mathrm{He}(M F)\right]$ and $\left[F_{2}^{d}(M F)\right] /\left[F_{2}^{d}(S R C)\right]$ (LC calculations are similar and can be found in the Appendices). As expected, mean-field nucleons account for most of the structure function in Eq. (A1), except at very high $x_{B}$ where nucleon motion effects are important and therefore the contribution of SRCs becomes significant. This is to be expected as SRC nucleons account for a small fraction of the nuclear wave function, especially in deuterium.

Next we explicitly examine the contribution of mean-field and SRC nucleons to the off-shell modification effect in the EMC. This is done by defining the off-shell decomposition as $F_{2}^{A}($ off-shell $)=F_{2}^{A}($ full $)-F_{2}^{A}($ no off-shell $)$, where $F_{2}^{A}($ full $)$ is calculated using Eq. (A1) and $F_{2}^{A}$ (no off-shell) is calculated using the same equation but by setting $f^{\text {off }}(\tilde{x})=0$.

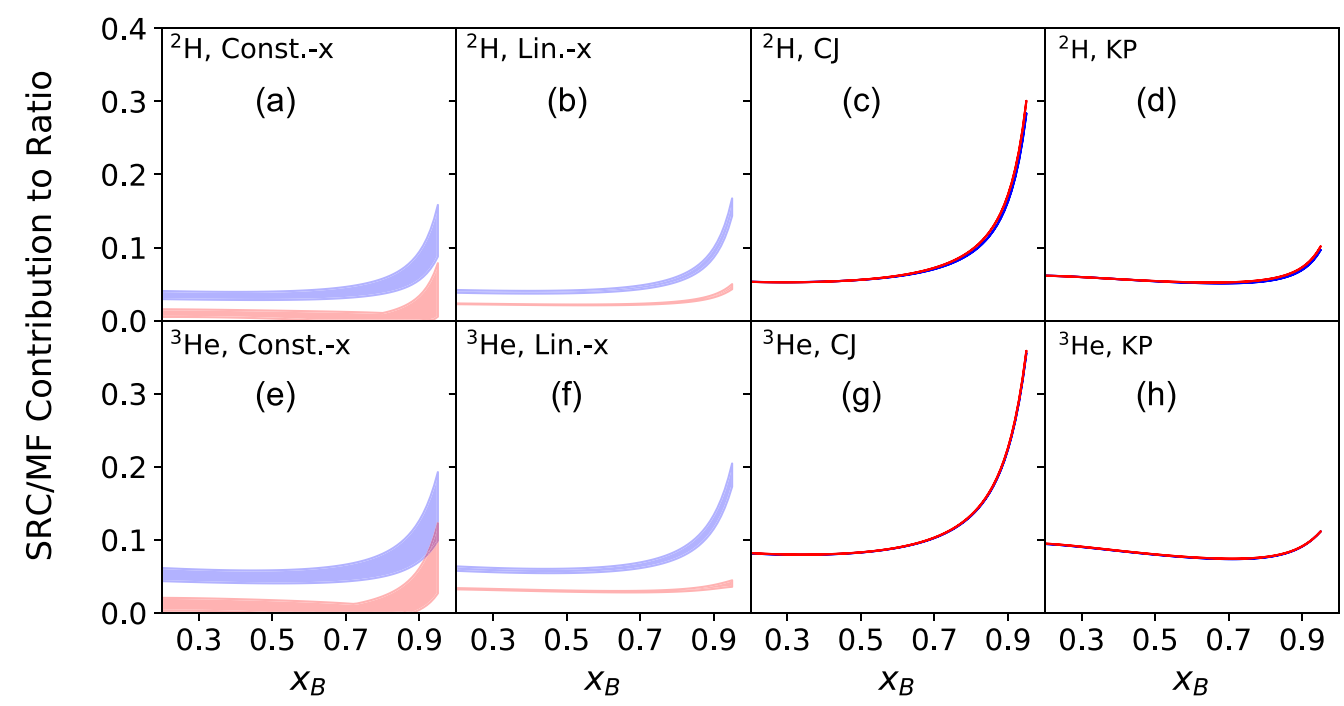

FIG. 6. Ratio of SRC contribution to MF contribution of the structure function using SF approximation. See text for details. [Top row, (a)-(d)] Ratio on $F_{2}^{d}$. [Bottom row, (e)-(h)] Ratio on $F_{2}^{3} \mathrm{He}$. In each row, results are shown for different off-shell functions used, in order from left to right: $f_{\text {const }}^{\text {off }}[(\mathrm{a}),(\mathrm{e})], f_{\operatorname{lin} x}^{\text {off }}[(\mathrm{b}),(\mathrm{f})], f_{C J}^{\text {off }}[(\mathrm{c}),(\mathrm{g})], f_{K P}^{\text {off }}[(\mathrm{d}),(\mathrm{h})] .1 \sigma$ confidence bands are displayed for each trial. Curves are shown with $Q^{2}=5 \mathrm{GeV}^{2}$. 


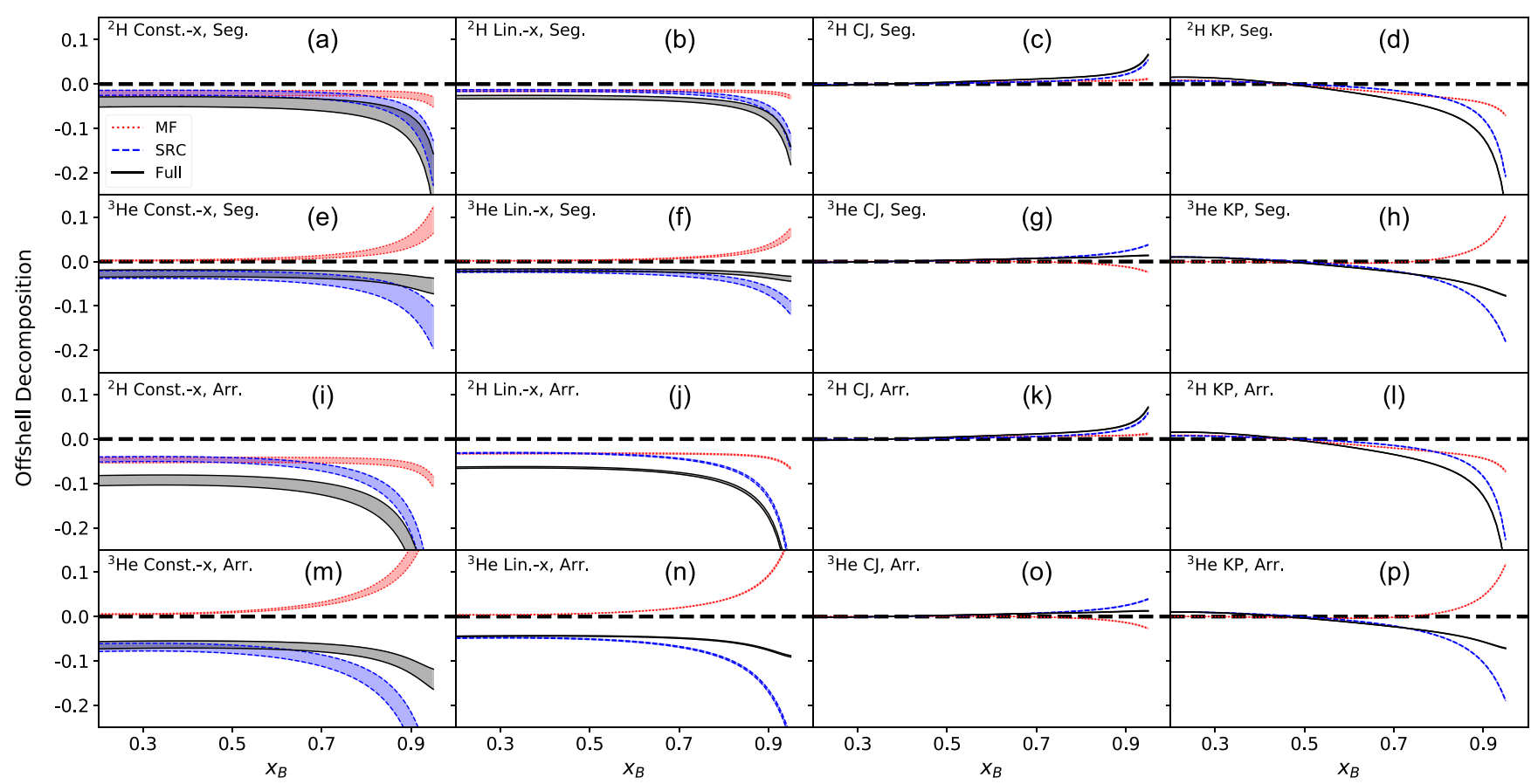

FIG. 7. Decomposition of $F_{2}^{A}$ (off-shell) for various model assumptions within the SF approximation. [Top row, (a)-(d)] Decomposition for $F_{2}^{d} /\left(F_{2}^{p}+F_{2}^{n}\right)$ with Segarra et al. $F_{2}^{n} / F_{2}^{p}$. [2nd row, (e)-(h)] Decomposition for $F_{2}^{3} H e / F_{2}^{d}$ with Segarra et al. $F_{2}^{n} / F_{2}^{p}$. [3rd row, (i)-(1)] Decomposition for $F_{2}^{d} /\left(F_{2}^{p}+F_{2}^{n}\right)$ with Arrington $F_{2}^{n} / F_{2}^{p}$. [Bottom row, (m)-(p)] Decomposition for $F_{2}^{3} H e / F_{2}^{d}$ with Arrington $F_{2}^{n} / F_{2}^{p}$. In each row, decompositions are shown for different off-shell functions used, in order from left to right: $f_{\text {const }}^{\text {off }}[(\mathrm{a}),(\mathrm{e}),(\mathrm{i}),(\mathrm{m})], f_{\text {lin } x}^{\text {off }}[(\mathrm{b}),(\mathrm{f}),(\mathrm{j}),(\mathrm{n})]$, $f_{C J}^{\text {off }}[(\mathrm{c}),(\mathrm{g}),(\mathrm{k}),(\mathrm{o})], f_{K P}^{\text {off }}[(\mathrm{d}),(\mathrm{h}),(\mathrm{l}),(\mathrm{p})]$. Solid black lines represent the full off-shell contribution. Dashed blue lines are the contribution due to SRC nucleons ( $>240 \mathrm{MeV} / \mathrm{c}$ in the SF assumption). Similarly, dotted red lines are the contribution due to MF nucleons ( $<240 \mathrm{MeV} / \mathrm{c}$ ). $1 \sigma$ confidence bands are displayed for each trial. Curves for GCF-LC approximation can be viewed in the Appendices. Curves are shown at $Q^{2}=5 \mathrm{GeV}^{2}$.

Figure 7 shows the decomposition of $F_{2}^{A}$ (off-shell) into $\mathrm{SRC}$ and mean-field nucleons within the SF approach (LC calculations are qualitatively similar and can be found in the

Appendices). While high-momentum nucleons did not significantly contribute to the full convolution ratio in Fig. 6, these nucleons dominate the off-shell modification function

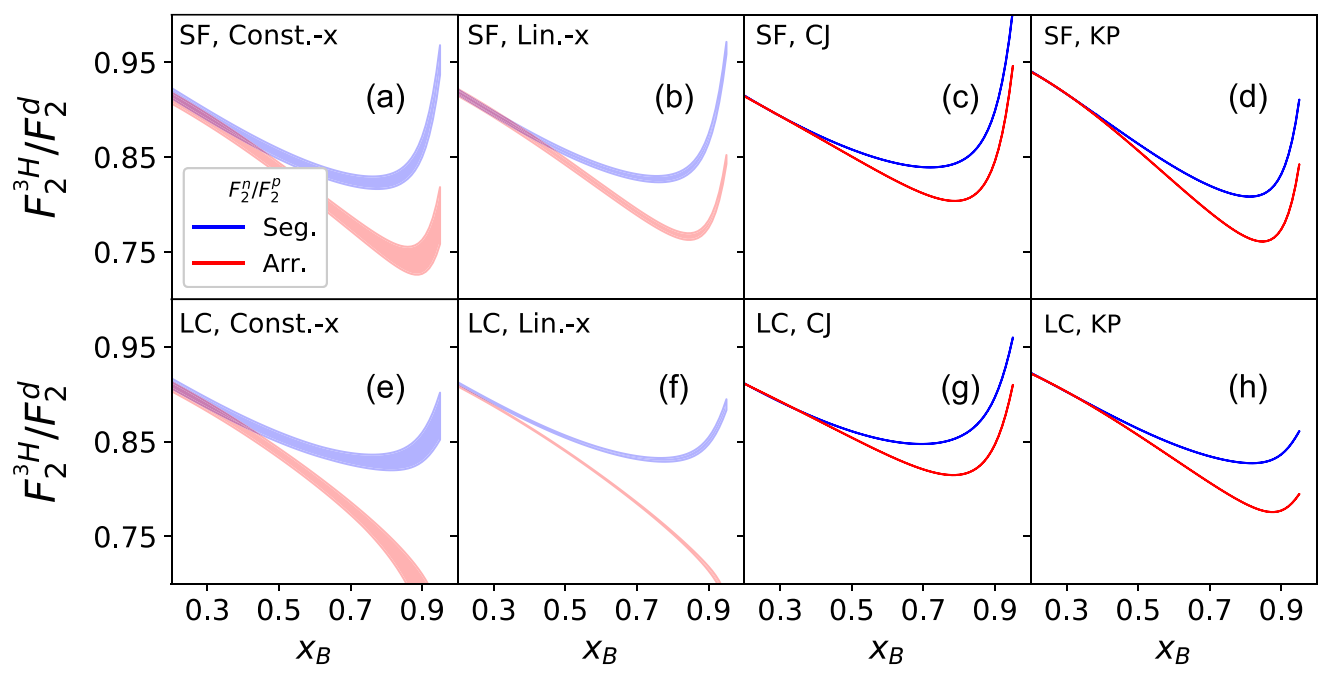

FIG. 8. Predictions of $\frac{F_{2}^{3} H}{F_{2}^{d}}$ using our convolution framework with the universal off-shell modification constrained from ${ }^{2} \mathrm{H}$ and ${ }^{3} \mathrm{He}$ data: [Top row, (a)-(d)] SF convolution, [Bottom row, (e)-(h)] GCF-LC convolution. In each row, results are shown for different off-shell functions used, in order from left to right: $f_{\text {const }}^{\text {off }}[(\mathrm{a}),(\mathrm{e})], f_{\text {lin } x}^{\text {off }}[(\mathrm{b}),(\mathrm{f})], f_{C J}^{\text {off }}[(\mathrm{c}),(\mathrm{g})], f_{K P}^{\text {off }}[(\mathrm{d}),(\mathrm{h})] .1 \sigma$ confidence bands are displayed for each prediction. See text for details. All curves are shown for MARATHON kinematics, i.e., $Q^{2}=14 \cdot x_{B}\left[\mathrm{GeV}^{2}\right]$. 


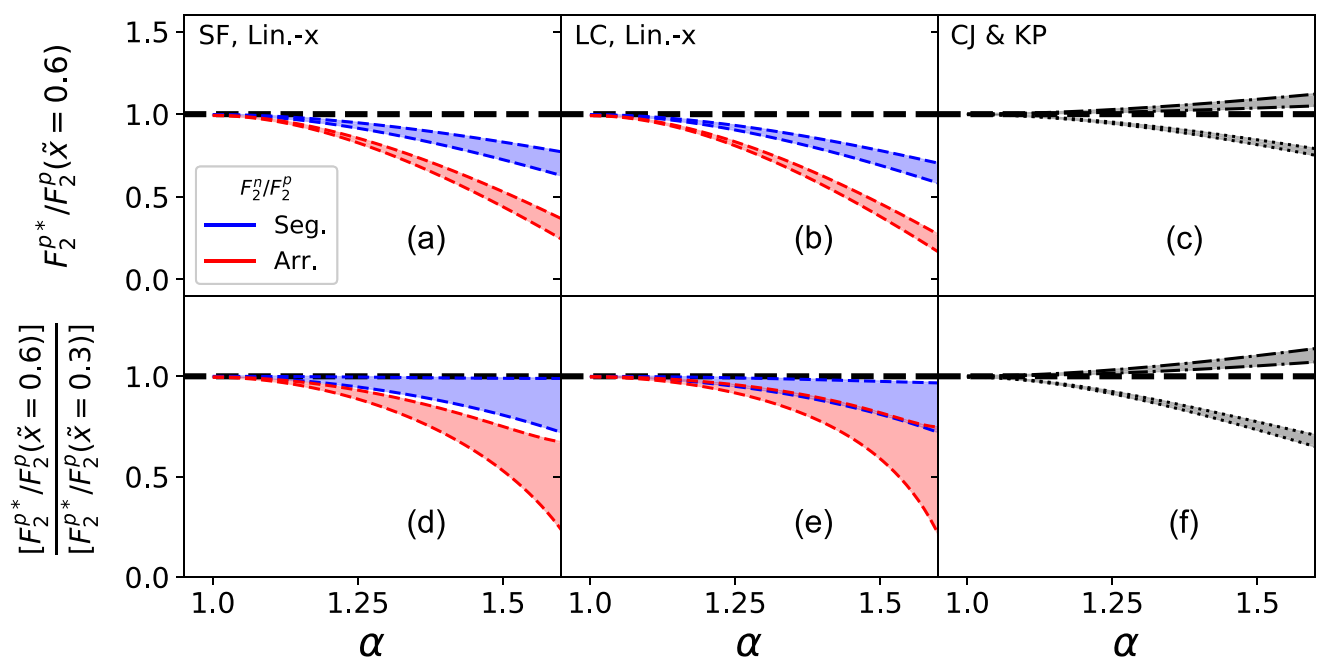

FIG. 9. [Top row, (a)-(c)] Predictions of the ratio of the bound proton structure function in deuterium to the free proton structure function as a function of $\alpha$ at a fixed $\tilde{x}=0.6$. [Bottom row, (d)-(f)] Predictions of the double ratio of bound-to-free proton structure function for $\tilde{x}=0.6$ to $\tilde{x}=0.3 .1 \sigma$ confidence bands are displayed for each prediction. In the bottom panels, predictions for models with $f_{\text {const }}^{\text {off }}$ yield a double ratio of 1 , as the modification is constant in $\tilde{x}$. See text for details. Curves are shown at $Q^{2}=5 \mathrm{GeV}^{2}$ and for $\mathbf{p}_{\mathbf{T}}=\mathbf{0}$. In (c) and (f), finely dotted lines use $f_{K P}^{\text {off }}$ and dashed-dotted lines use $f_{C J}^{\text {off }}$.

(i.e., the dashed blue lines track the solid black lines closely, especially at high $x_{B}$ ) in all models even though the off-shell behavior is different for each model.

This holds true even in deuterium at high $x_{B}$, although at $x_{B} \sim 0.6$, the mean-field and SRC contributions are closer to $1: 1$. This is still surprising given the high-momenta fraction of the nuclear momentum-distribution is only $O(\sim 4 \%)$ [51]. Adding to this surprise is the feature that a significant contribution to the wave function comes from $n p$ separations larger than the range of the nuclear forces [52].

Furthermore, in the results shown here using the SF approach, the momentum sum rule is violated by $\sim 1 \%$. While small, this violation still induces an artificial EMC effect, thereby reducing the strength of the actual off-shell contribution to the structure function (i.e., the absolute $y$ scale of Fig. 7). Alternatively, in the LC approach, the sum rules are manifestly satisfied, and the extracted off-shell contribution is much larger for the models of $F_{2}^{n} /\left.F_{2}^{p}\right|_{\text {Seg. }}$ by a factor of about $1.5-3$ (see Appendices).

Our findings are robust to the exact underlying off-shell function used in Eq. (A1), even though $f^{\text {off }}(\tilde{x})$ (Fig. 5) varies dramatically among the models. Therefore, the results shown in Fig. 7 contradict the recent claims of Ref. [53], where the SRC UMF was analyzed without proper separating its contributions from nucleon motion and modification effects. For completeness we note that the UMF extracted by Ref. [7,21] is reproduced with the convolution framework used here for ${ }^{3} \mathrm{He}$, see Appendices.

\section{PREDICTING FORTHCOMING OBSERVABLES}

While existing data cannot constrain $F_{2}^{n} / F_{2}^{p}$, here we show predictions $F_{2}^{3} H / F_{2}^{d}$, which was recently measured by the MARATHON collaboration [24], and should be sensitive to $F_{2}^{n}$. Figure 8 shows the convolution prediction for $F_{2}^{3} H / F_{2}^{d}$ obtained using the constrained off-shell modification function and assuming isospin symmetry in the lightcone distributions. The different $F_{2}^{n} / F_{2}^{p}$ parametrizations, which are both consistent with $F_{2}^{3} \mathrm{He} / F_{2}^{d}$ data, predict very different $F_{2}^{3} \mathrm{H} / F_{2}^{d}$ at high $x_{B}$ that MARATHON can test. Still, as seen in Fig. 8, there are predictions of $F_{2}^{3} H / F_{2}^{d}$, which overlap for very
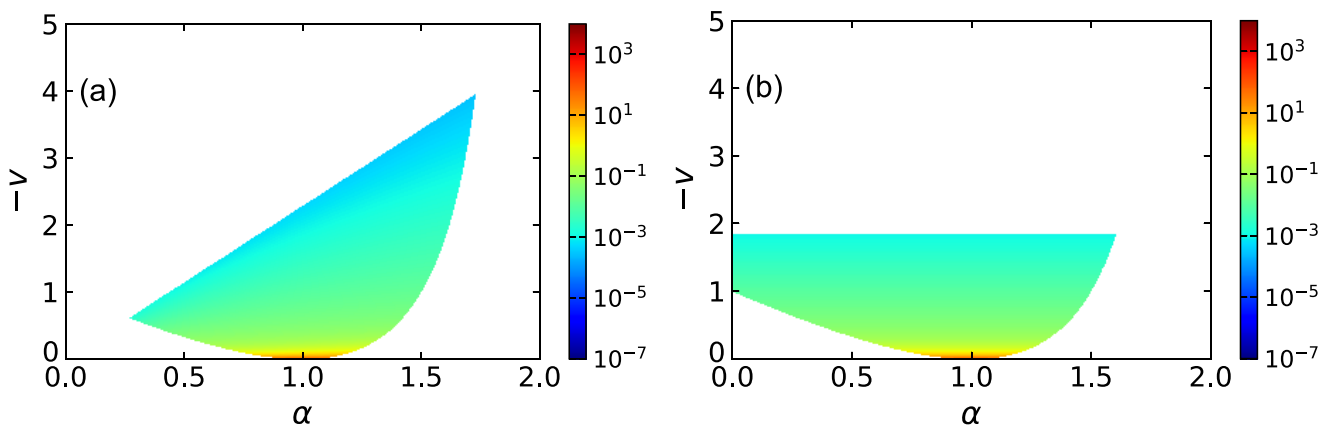

FIG. 10. Comparison of $\tilde{\rho}_{p, n}^{2}(\alpha, v)$ for the (a) GCF-LC formalism and (b) SF formalism. Due to isospin symmetry, the distributions are identical for proton and neutron. 

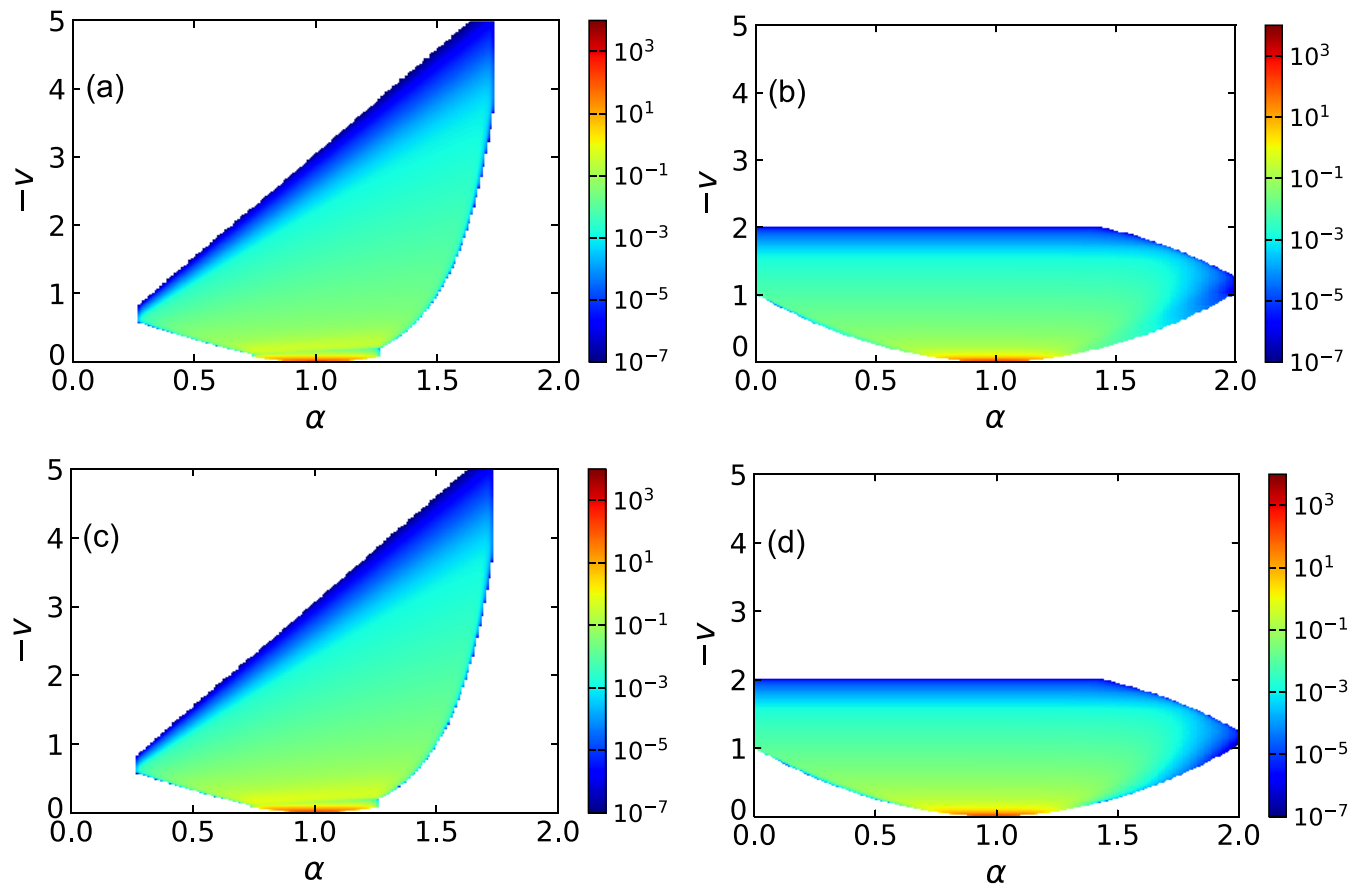

FIG. 11. Comparison of $\tilde{\rho}_{p, n}^{3} \mathrm{He}(\alpha, v)$ for the (a), (c) GCF-LC formalism and (b), (d), SF formalism. Proton distributions are shown above $[(\mathrm{a}),(\mathrm{b})]$ and neutron distributions are shown below $[(\mathrm{c}),(\mathrm{d})]$.

different $F_{2}^{n} / F_{2}^{p}$ and $f^{\text {off }}$ behaviors. In particular, taking the $f_{K P}^{\text {off }}$ with $F_{2}^{n} /\left.F_{2}^{p}\right|_{\text {Seg. }}$ (blue dotted) and $f_{C J}^{\text {off }}$ with $F_{2}^{n} /\left.F_{2}^{p}\right|_{\text {Arr. }}$ (red dash-dotted), yield overlapping predictions. This indicates a combined analysis of nuclear DIS data with forthcoming data by MARATHON will be needed to disentangle $F_{2}^{n} / F_{2}^{p}$ and $f^{\text {off }}$, similar to efforts Ref. [22] has performed in the past.

While the MARATHON results will be very sensitive to $F_{2}^{n} / F_{2}^{p}$, they will be less sensitive to the exact nature of the offshell modification function ( $\left.f^{\text {off }}\right)$. This can however be tested in a new set of tagged deep inelastic scattering measurements off deuterium [25,26,54], which will study the dependence of the bound nucleon structure function on $\alpha$, see Fig. 9:

$$
F_{2}^{p *}(\tilde{x}, \alpha)=F_{2}^{p}(\tilde{x})\left[1+\left.\langle v\rangle\right|_{\alpha} f^{\text {off }}(\tilde{x})\right],
$$

where $\left.\langle v\rangle\right|_{\alpha}$ is the average fractional virtuality for the given $\alpha$, see Figs. 10 and 11 for the phase space of the lightcone densities.

By taking a ratio of the bound-to-free proton structure function, one can access the off-shell modification function, and can examine the differences of the off-shell contribution at high $\tilde{x}$ and low $\tilde{x}$, see Fig 9. The predictions here are similar to those made by Ref. [54] and will be directly tested by the LAD [26] and BAND [25] Collaborations. The latter already completed $50 \%$ data taking and results are anticipated soon. While predictions here are made for $\mathbf{p}_{\mathbf{T}}=\mathbf{0}$, experiments will have some finite acceptance in $\mathbf{p}_{\mathbf{T}}$. As seen in Fig 9, there are significant uncertainties due to uncertainties in $F_{2}^{n} / F_{2}^{p}$ (red vs blue curves). However, after precise measurements on $F_{2}^{n} / F_{2}^{p}$ by the MARATHON Collaboration [24], these uncertainties will be greatly reduced.

\section{SUMMARY}

We present an extensive study of nucleon modification effects in nuclei using a convolution formalism and measurements of the EMC effect in deuterium and ${ }^{3} \mathrm{He}$. We examine a range of off-shell modification functions, free-neutron structure function models, and different treatments of nucleon motion effects. In all cases we find that nucleons in SRC pairs are the dominant contribution to nucleon modification effects in deuterium and ${ }^{3} \mathrm{He}$.

With upcoming precise measurements of ${ }^{3} \mathrm{H}$, our study can be extended to test the isospin dependence of the universal off-shell modification function and the ability to use nuclear DIS data to constrain the free neutron structure function. We stress that an isospin-dependent EMC effect, in the sense of a different average modification for protons and neutrons, as, e.g., suggested by Refs. [11,12,55,56], can be obtained in all models discussed in this paper if the proton and neutron lightcone densities have different average virtualities. In addition we make predictions for new measurements of the bound nucleon structure function. These measurements will allow us to further constrain the elements of our model.

\section{ACKNOWLEDGMENTS}

This work was supported by the U.S. Department of Energy, Office of Science, Office of Nuclear Physics under Awards No. DE-FG02-94ER40818, No. DE-FG02-96ER-40960, No. DE-AC05-06OR23177, No. DE-FG02- 93ER-40771, and No. DE-FG02-97ER-41014 under which Jefferson Science Associates operates the Thomas Jefferson National Accelerator Facility, the Pazy foundation, and the Israeli Science Foundation (Israel) under Grants No. 136/12 and No. 1334/16. 

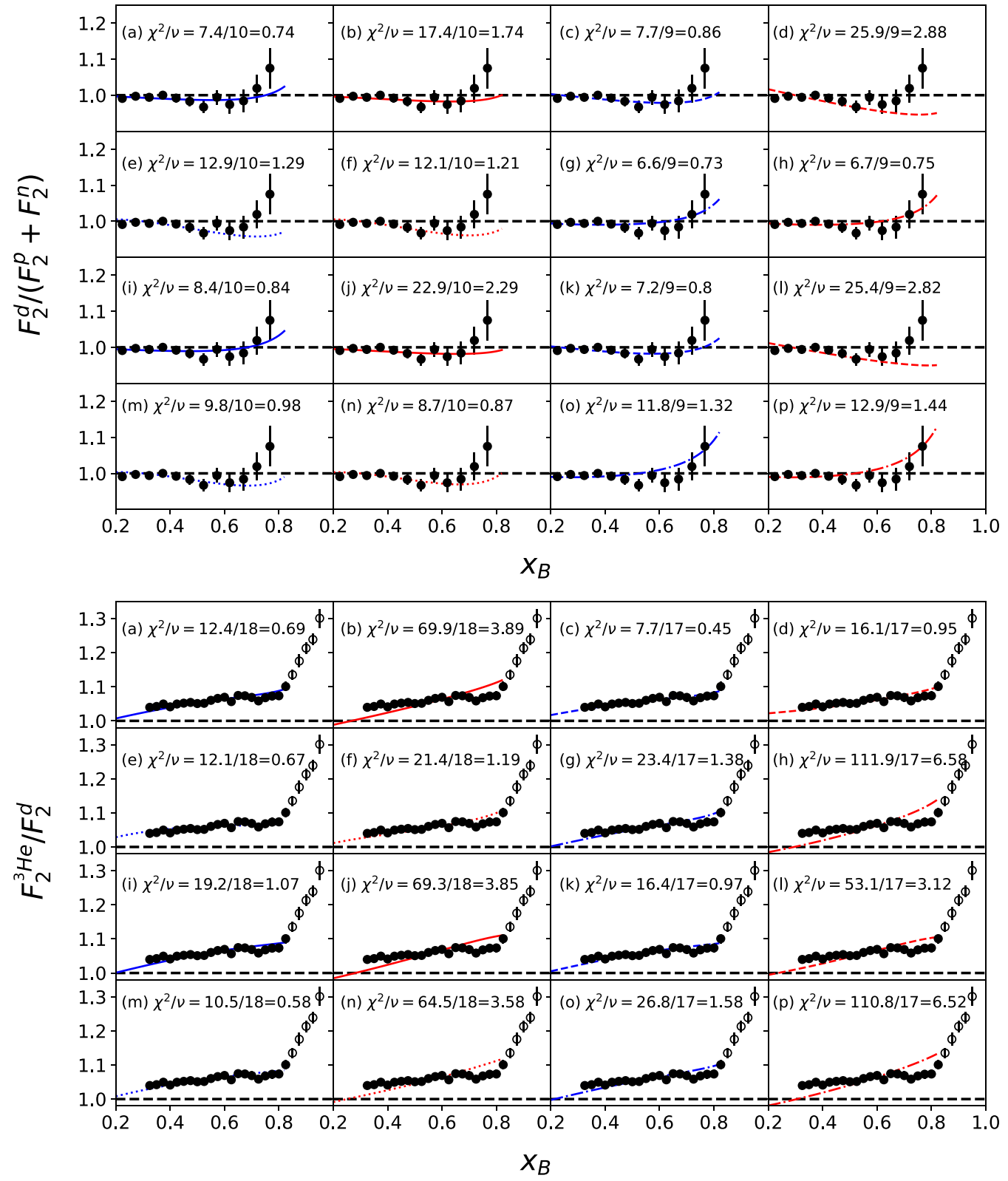

FIG. 12. Labels for subplots signify fit results using model assumptions described in $\chi^{2}$-minimization procedure. (Top): fit quality to $F_{2}^{d} /\left(F_{2}^{p}+F_{2}^{n}\right)$ data. (Bottom): fit quality to $F_{2}^{3} \mathrm{He} / F_{2}^{d}$ data.

\section{APPENDIX A: $\chi^{2}$-MINIMIZATION PROCEDURE}

We calculate $F_{2}^{A}\left(x_{B}\right)$ for ${ }^{3} \mathrm{He}$ and ${ }^{2} \mathrm{H}$ in a nuclear convolution approximation:

$$
\begin{aligned}
& F_{2}^{A}\left(x_{B}, Q^{2}\right)=\int_{x_{B}}^{A} \frac{d \alpha}{\alpha} \int_{-\infty}^{0} d v F_{2}^{p}\left(\tilde{x}, Q^{2}\right) \\
& \times\left[Z \tilde{\rho}_{p}^{A}(\alpha, v)+N \tilde{\rho}_{n}^{A}(\alpha, v) \frac{F_{2}^{n}}{F_{2}^{p}}(\tilde{x})\right]\left(1+v f^{\text {off }}(\tilde{x})\right),
\end{aligned}
$$

and form the EMC ratios of $F_{2}^{3} \mathrm{He} / F_{2}^{d}$ to compare to [6] and $F_{2}^{d} /\left(F_{2}^{p}+F_{2}^{n}\right)$ to compare to [49] in order to infer parameters of interest (see below Sec. D on low $Q^{2}$, high- $x_{B}$ sensitivity for definition of $\tilde{x}$ and discussion of finite- $Q^{2}$ effects). In particular, we would like to investigate the nucleon off-shell modification function $f^{\text {off }}(\tilde{x})$, which we assume to be the same for neutrons and protons, as well as the same across all nuclei. The nucleon off-shell modification is assumed to be linear in virtuality $v=\frac{E^{2}-p^{2}-m_{N}^{2}}{m_{N}^{2}}$ (hence the $[1+v f]$ form). We consider three models:

$$
\begin{aligned}
f_{\text {const }}^{\text {off }}(\tilde{x}) & =a_{\text {const }}, \\
f_{\operatorname{lin} x_{B}}^{\text {off }}(\tilde{x}) & =a_{\operatorname{lin} x_{B}}+b_{\operatorname{lin} x_{B}} \cdot \tilde{x} \\
f_{K P, C J}^{\text {off }}(\tilde{x}) & =C\left(\tilde{x}-x_{0}\right)\left(\tilde{x}-x_{1}\right)\left(1+x_{0}-\tilde{x}\right)
\end{aligned}
$$

To perform the parameter inference, we first construct the structure function ratios [following Eq. (A1)] for each kinematic point $i\left(x_{B}, Q^{2}\right)$ in the data sets considered, i.e., $\left.\frac{F_{2}^{3^{3} H e}}{F_{2}^{d}}\right|_{i, t h e o}$ and $\left.\frac{F_{2}^{d}}{F_{2}^{p}+F_{2}^{n}}\right|_{i, t h e o}$ using initial guesses on the parameters of interest. We also allow for a floating renormalization of the 


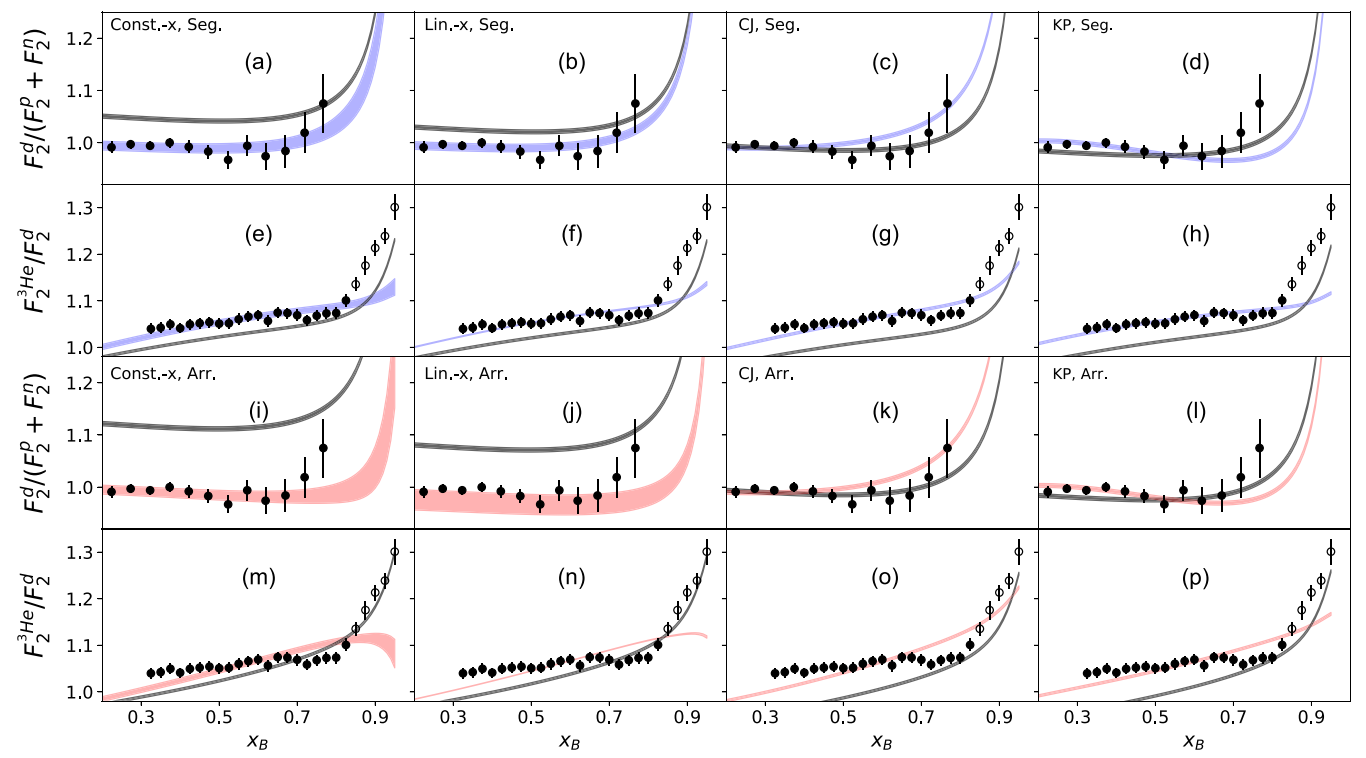

FIG. 13. Equivalent figure as Fig. 4 in the main text, but for the lightcone convolution approximation. Convolution results after $\chi^{2}-$ minimization procedure. $1 \sigma$ confidence bands are displayed for each trial, and each band is shown with the renormalization inferred for ${ }^{2} \mathrm{H}$ and ${ }^{3} \mathrm{He}$. The result of no off-shell modification (grey bands) are also shown with the renormalization preferred for that given fit. The data used in the minimization $\left({ }^{2} \mathrm{H}\right.$ data from [49] and ${ }^{3} \mathrm{He}$ data of [6]) are shown as filled circles. Open circles denote data at $W<1.4 \mathrm{GeV}$, which were not used in the fit. [Top row, (a)-(d)] results for $F_{2}^{d} /\left(F_{2}^{p}+F_{2}^{n}\right)$ with Segarra et al. $F_{2}^{n} / F_{2}^{p}$. [2nd row, (e)-(h)] Results for $F_{2}^{3} H e / F_{2}^{d}$ with Segarra et al. $F_{2}^{n} / F_{2}^{p}$. [3rd row, (i)-(1)] Results for $F_{2}^{d} /\left(F_{2}^{p}+F_{2}^{n}\right)$ with Arrington $F_{2}^{n} / F_{2}^{p}$. [Bottom row, (m)-(p)] Results for $F_{2}^{3} H e / F_{2}^{d}$ with Arrington $F_{2}^{n} / F_{2}^{p}$. In each row, results are shown for different off-shell functions used, in order from left to right: $f_{\text {const }}^{\text {off }}[(\mathrm{a}),(\mathrm{e}),(\mathrm{i}),(\mathrm{m})], f_{\text {lin } x}^{\text {off }}$ $[(\mathrm{b}),(\mathrm{f}),(\mathrm{j}),(\mathrm{n})], f_{C J}^{\text {off }}[(\mathrm{c}),(\mathrm{g}),(\mathrm{k}),(\mathrm{o})], f_{K P}^{\text {off }}[(\mathrm{d}),(\mathrm{h}),(\mathrm{l}),(\mathrm{p})]$. All curves are calculated and extrapolated with the same $Q^{2}$ as the data for ${ }^{2} \mathrm{H}$ and ${ }^{3} \mathrm{He}$.

data sets, $N_{B}$ for [49] and $N_{S}$ for [6]. We then calculate a $\chi^{2}$ for the current parameter assumptions $\left(f^{\text {off }}(\tilde{x}), N_{B}, N_{S}\right)$ :

$$
\begin{aligned}
\chi^{2}= & \sum_{i \in \in^{2} H}\left(\frac{\left.N_{B} \cdot \frac{F_{2}^{d}}{F_{2}^{p}+F_{2}^{n}}\right|_{i, \text { theo }}-\left.\frac{F_{2}^{d}}{F_{2}^{p}+F_{2}^{n}}\right|_{i, \text { meas }}}{\sigma_{i}}\right)^{2} \\
& +\sum_{i \in \in^{3} \mathrm{He}}\left(\frac{N_{S} \cdot \frac{F_{2}^{3}{ }_{F_{2}}{ }^{\mathrm{He}}}{{ }_{i, \text { theo }}-\left.\frac{F_{2}{ }^{3} \mathrm{He}}{F_{2}^{d}}\right|_{i, \text { meas }}}}{\sigma_{i}}\right)^{2}
\end{aligned}
$$

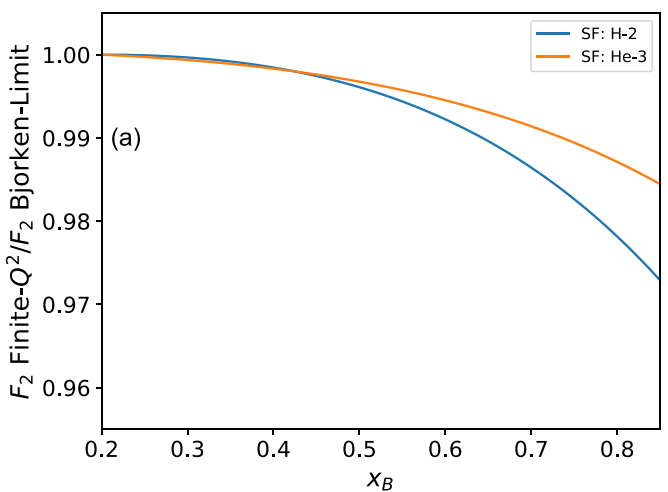

$$
+\left(\frac{N_{B}-1}{\sigma_{\mathrm{norm}, B}}\right)^{2}+\left(\frac{N_{S}-1}{\sigma_{\mathrm{norm}, S}}\right)^{2},
$$

and minimize $\chi^{2}$ to infer our parameters utilizing Minuit2 MIGRAD algorithm. $F_{2}^{p}\left(\tilde{x}, Q^{2}\right)$ carries the only $Q^{2}$ dependence we assume in Eq. (A1). We calculated $F_{2}^{p}$ using the GD11-P parameterization [48]. We also performed a study utilizing the Bosted-Christy [50] parameterizations of $F_{2}^{p}, F_{2}^{n}$ to investigate resonance contributions (see below). $\tilde{\rho}_{p, n}^{A}(\alpha, v)$ are the lightcone distributions for protons and neutrons in a given nucleus $A$. The minimization procedure was performed

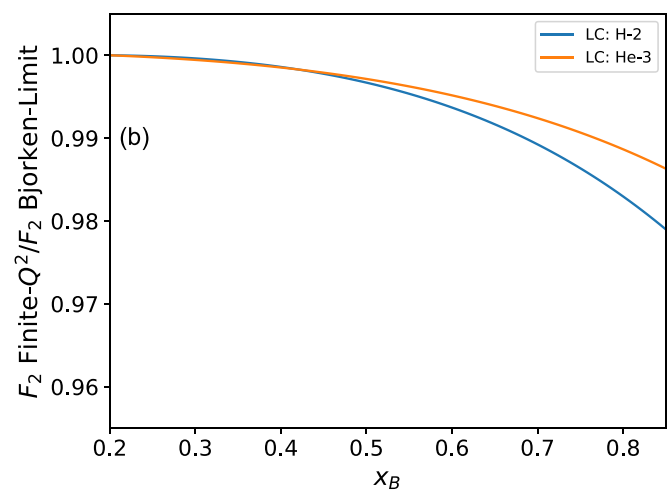

FIG. 14. Ratio of full (finite- $Q^{2}$ ) convolution integral to Bjorken-limit convolution integral. In blue, ratio of $\left.F_{2}^{d}\right|_{\text {finite }}$ to $\left.F_{2}^{d}\right|_{\text {Bjorken-limit }}$ is shown. In orange, double ratio of $\left[\left.F_{2}^{3} H e\right|_{\text {finite }} /\left.F_{2}^{d}\right|_{\text {finite }}\right]$ to $\left[\left.F_{2}^{3} H e\right|_{\text {Bjorken-limit }} /\left.F_{2}^{d}\right|_{\text {Bjorken-limit }}\right]$ is shown. (a) Spectral function convolution approximation. (b) Lightcone convolution approximation. To interpolate over a wide range of $x_{B}$ values, a parameterization of the $Q^{2}\left(x_{B}\right)$ reach of each experiments (Deuterium and Helium-3 separately) was utilized. 

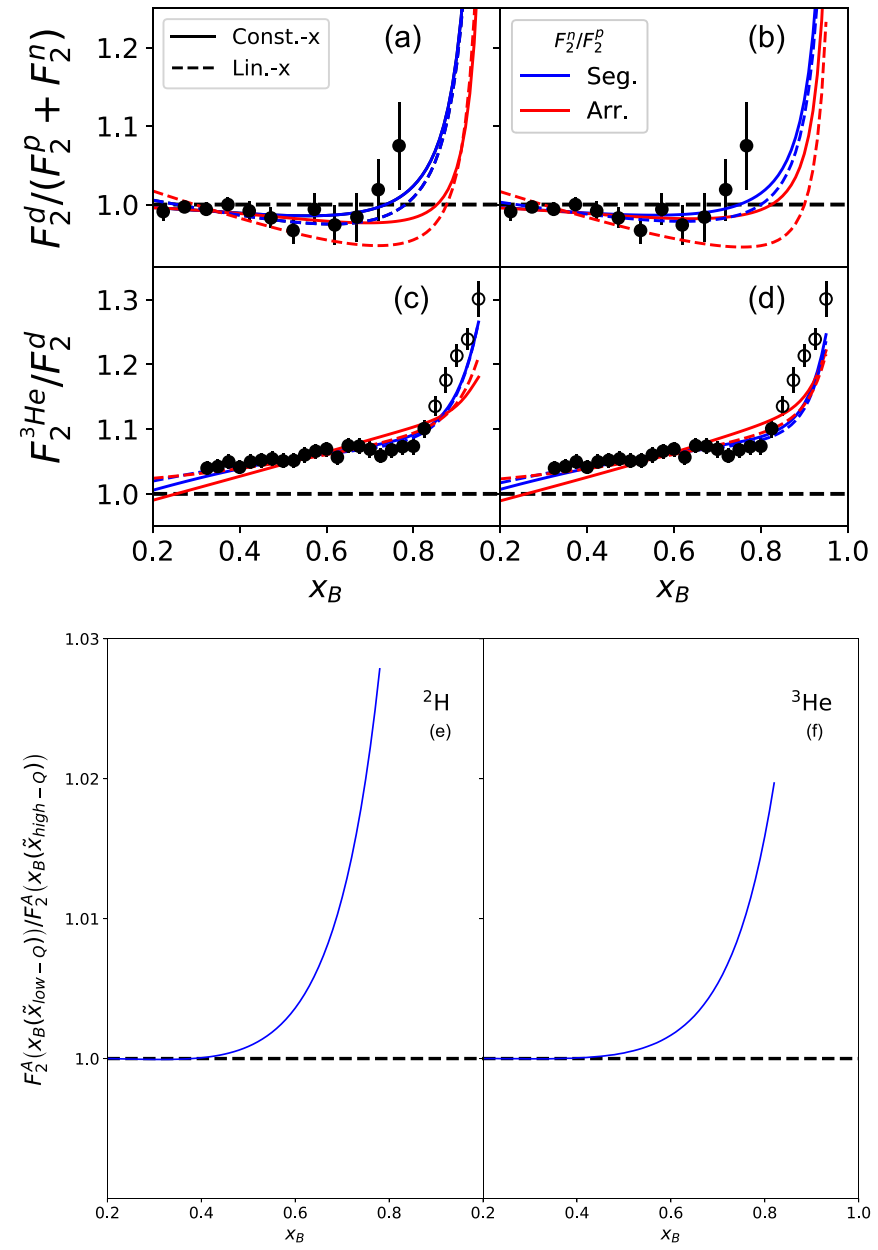

FIG. 15. [Top, (a)-(d)] Convolution results using the finiteenergy expression of Eq. (D2) for $\tilde{x}$ (Low-Q Convolution) and Bjorken frame of Eq. (D3) for $\tilde{x}$ (High-Q Convolution) for deuterium and Helium-3. [Bottom, (e),(f)] The ratio of structure functions $F_{2}^{A}\left(x_{B}\right)$ calculated with Eq. (D2) to that calculated with Eq. (D3). While at high $x_{B}$, the ratio starts to diverge above $2 \%$, we only consider data up to $x_{B} \sim 0.8$.

two times: using a spectral function approximation to $\tilde{\rho}(\alpha, v)$ and using the lightcone formalism (see main text for details).

$F_{2}^{n} / F_{2}^{p}$ is parameterized as $F_{2}^{n} / F_{2}^{p}(\tilde{x})=a_{n p}(1-\tilde{x})^{b_{n p}}+$ $c_{n p}$, where there is no explicit $Q^{2}$ considered, except for the study with the Bosted-Christy parameterizations (see below). We fit this parameterization to two recent predictions by Segarra [21] and Arrington [47], and then perform the $\chi^{2}$ minimization using each $F_{2}^{n} / F_{2}^{p}$ prediction. We do not allow parameter variation of $F_{2}^{n} / F_{2}^{p}$ in the minimization procedure with the two data sets considered. However, by taking two extreme predictions of $F_{2}^{n} / F_{2}^{p}$, we can study the sensitivity our results have to the exact nature of $F_{2}^{n} / F_{2}^{p}$.

In the case of using $f_{K P, C J}^{\text {off }}(\tilde{x})$, we fixed the parameters of the off-shell function to those found in a minimization by [22] $\left(f_{K P}^{\text {off }}\right)$ and by [46] $\left(f_{C J}^{\text {off }}\right)$. We note that while the same off-shell parameterization is used by both groups, Ref. [22] considered a wide range of nuclear DIS data sets and [46] used DIS data only on the proton and deuterium to infer their off-shell function.

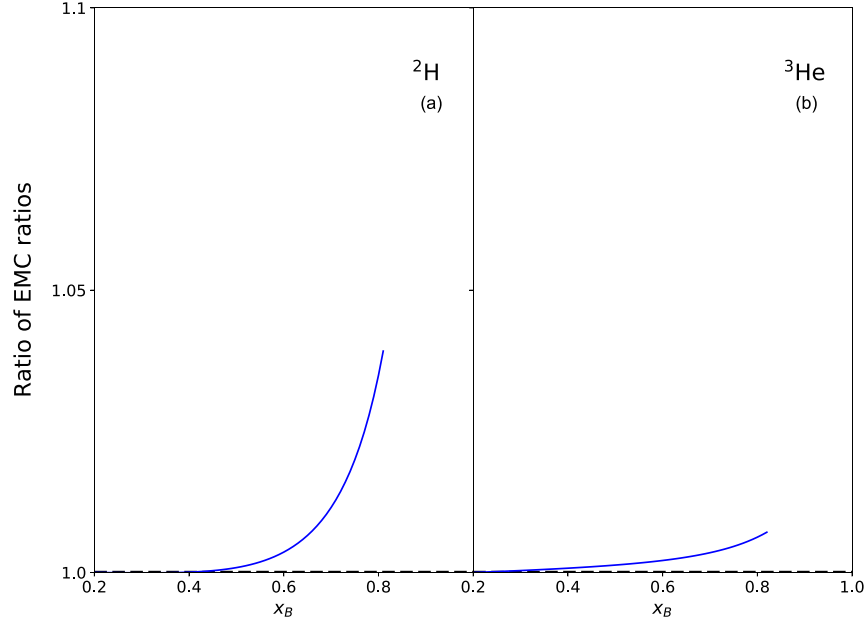

FIG. 16. The ratio of EMC ratios calculated with Eq. (E1) to that calculated with Eq. (D3). (a) The ratio of $\left.\left(F_{2}^{d} /\left(F_{2}^{n}+F_{2}^{p}\right)\right)\right|_{\mathrm{Eq} \text {.E1 }} /\left.\left(F_{2}^{d} /\left(F_{2}^{n}+F_{2}^{p}\right)\right)\right|_{\mathrm{Eq} . \mathrm{D} 3}$ and (b) the ratio of $\left.\left(F_{2}^{3} \mathrm{He} / F_{2}^{d}\right)\right|_{\mathrm{Eq} . \mathrm{E} 1} /\left.\left(F_{2}^{3} \mathrm{He} / F_{2}^{d}\right)\right|_{\mathrm{Eq} . \mathrm{D} 3}$.

Sixteen minimization trials were presented in the main text for the various model assumptions:
(a) SF with $F_{2}^{n} /\left.F_{2}^{p}\right|_{\text {Seg. }}$ with $f_{\text {const }}^{\text {off }}$
(b) SF with $F_{2}^{n} /\left.F_{2}^{p}\right|_{\text {Arr. }}$ with $f_{\text {const }}^{\text {off }}$
(c) SF with $F_{2}^{n} /\left.F_{2}^{p}\right|_{\text {Seg. }}$ with $f_{\operatorname{lin} x_{B}}^{\text {off }}$
(d) SF with $F_{2}^{n} /\left.F_{2}^{p}\right|_{\text {Arr. }}$ with $f_{\operatorname{lin} x_{B}}^{\text {off }}$
(e) SF with $F_{2}^{n} /\left.F_{2}^{p}\right|_{\text {Seg. with } f_{K P}^{\text {off }}}$
(f) $\mathrm{SF}$ with $F_{2}^{n} /\left.F_{2}^{p}\right|_{\text {Arr. }}$ with $f_{K P}^{\text {off }}$
(g) SF with $F_{2}^{n} /\left.F_{2}^{p}\right|_{\text {Seg. with } f_{C J}^{\text {off }}}$
(h) $\mathrm{SF}$ with $F_{2}^{n} /\left.F_{2}^{p}\right|_{\text {Arr. }}$ with $f_{C J}^{\text {off }}$
(i) GCF-LC with $F_{2}^{n} /\left.F_{2}^{p}\right|_{\text {Seg. with }} f_{\text {const }}^{\text {off }}$
(j) GCF-LC with $F_{2}^{n} /\left.F_{2}^{p}\right|_{\text {Arr. }}$ with $f_{\text {const }}^{\text {off }}$
(k) GCF-LC with $F_{2}^{n} /\left.F_{2}^{p}\right|_{\text {Seg. }}$ with $f_{\text {lin } x_{L}}^{\text {off }}$
(1) GCF-LC with $F_{2}^{n} /\left.F_{2}^{p}\right|_{\text {Arr. }}$ with $f_{\operatorname{lin} x_{B}}^{\text {off }}$
(m) GCF-LC with $F_{2}^{n} /\left.F_{2}^{p}\right|_{\text {Seg. }}$ with $f_{K P}^{\text {off }}$
(n) GCF-LC with $F_{2}^{n} /\left.F_{2}^{p}\right|_{\text {Arr. }}$ with $f_{K P}^{\text {off }}$
(o) GCF-LC with $F_{2}^{n} /\left.F_{2}^{p}\right|_{\text {Seg. }}$ with $f_{C J}^{\text {off }}$
(p) GCF-LC with $F_{2}^{n} /\left.F_{2}^{p}\right|_{\text {Arr. }}$ with $f_{C J}^{\text {off }}$

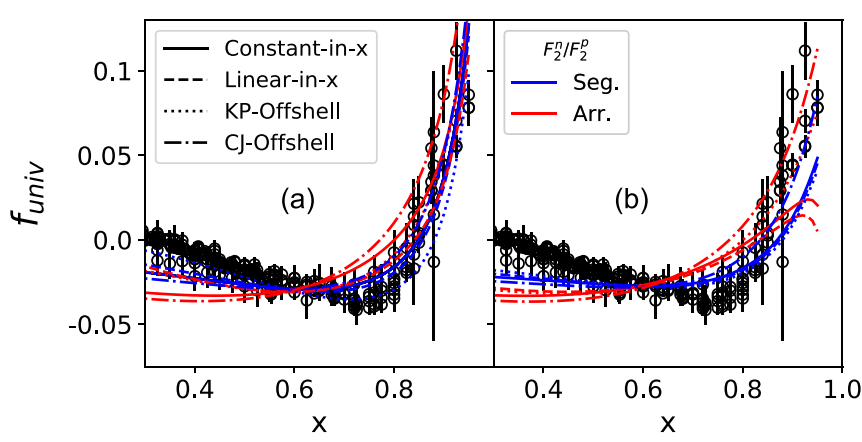

FIG. 17. Convolution results for ${ }^{3} \mathrm{He}$ shown as the universal modification function as described in [21], which was unable to decompose nucleon off-shell and motion effects. All curves are calculated and extrapolated with the same $Q^{2}$ as the data for ${ }^{3} \mathrm{He}$. (a) Uses the spectral function approach, and (b) uses the lightcone approach to the convolution. 

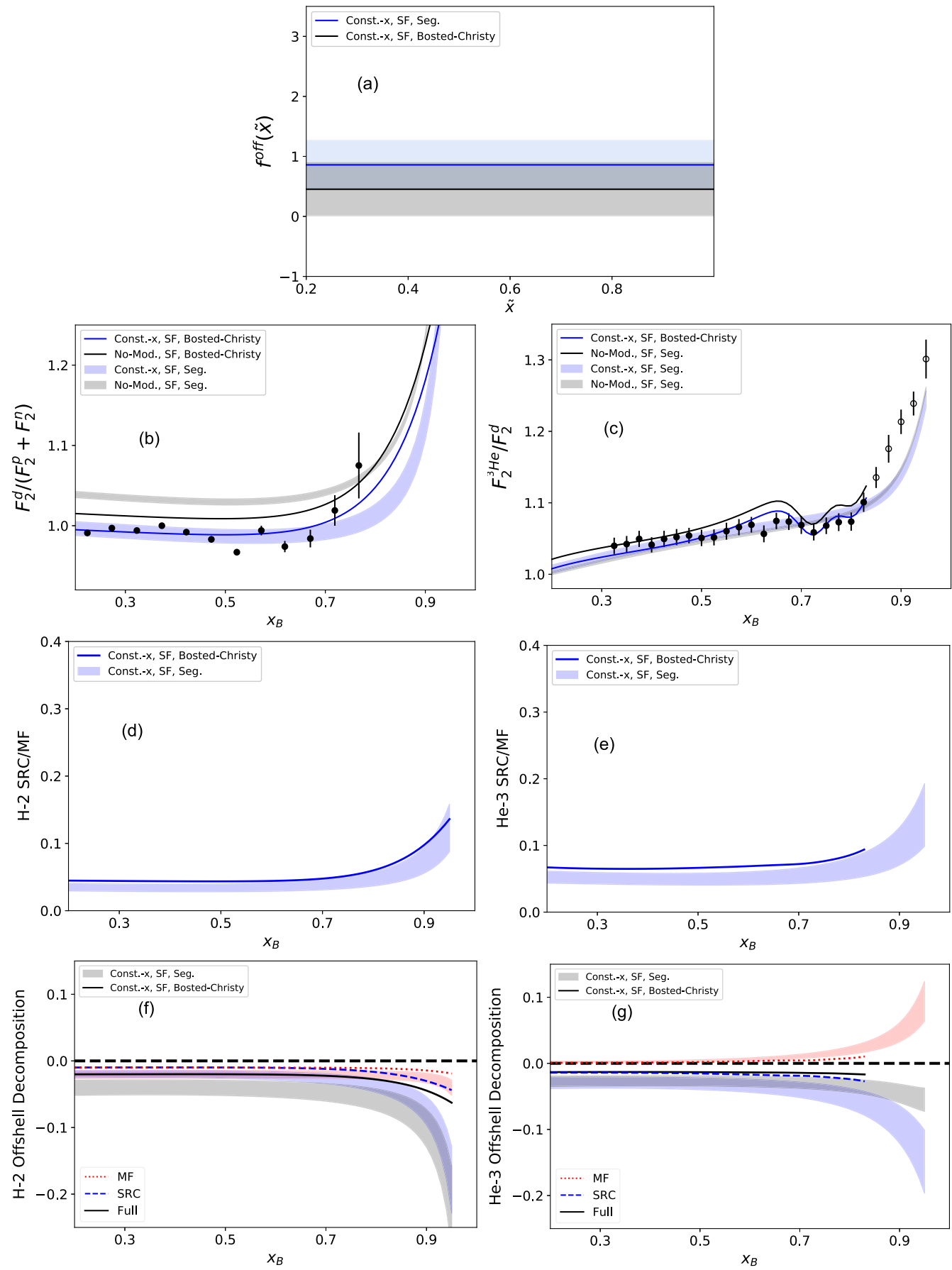

FIG. 18. Minimization results and analysis conclusions utilizing the Bosted-Christy parameterizations of $F_{2}^{p}$ and $F_{2}^{n}$ (solid lines) compared to a minimization trial with $F_{2}^{n} / F_{2}^{p}$ from Segarra [21] (shaded bands). Both trials used an off-shell function that was constant in $x, f_{\text {const }}^{\text {off }}$, and the spectral function approximation to the convolution. As was found when using $F_{2}^{n} / F_{2}^{p}$ from Arrington [47], the resulting off-shell function extracted depends on the underlying neutron structure assumed. However, universal features still emerge, such as the MF dominance to the full structure function, and the SRC dominance of the off-shell effect. (a) Off-shell function extracted from minimization trial. (b) Calculation of $F_{2}^{d} /\left(F_{2}^{p}+F_{2}^{n}\right)$ with resulting parameters compared to BONUS data. (c) Calculation of $F_{2}^{3} H e / F_{2}^{d}$ with resulting parameters compared to Seely data. (d) SRC/MF fraction of $F_{2}^{d}$. (e) SRC/MF fraction of $F_{2}^{3} H e$. (f) off-shell contributions to $F_{2}^{d}$. (g) Off-shell contributions to $F_{2}^{3} H e$.

where $\mathrm{SF}$ is a minimization trial that approximates $\tilde{\rho}(\alpha, v)$ with a spectral function, GCF-LC is a minimization trial that uses the lightcone formalism for $\tilde{\rho}(\alpha, v), F_{2}^{n} /\left.F_{2}^{p}\right|_{\text {seg. is a min- }}$ imization trial that uses $F_{2}^{n} / F_{2}^{p}$ fitted to [21], and $F_{2}^{n} /\left.F_{2}^{p}\right|_{\text {Arr. }}$ uses a fit to [47]. We performed and present additional iterations to investigate finite- $Q^{2}$ effects (see below).

\section{APPENDIX B: SF AND GCF-LC COMPARISON}

The distributions $\tilde{\rho}(\alpha, v) / \alpha$ of lightcone fraction and virtuality as shown below for deuterium in Fig. 10 and helium-3 in Fig. 11, using both the GCF lightcone formalism (GCFLC) and the spectral function formalism (SF). We note that 

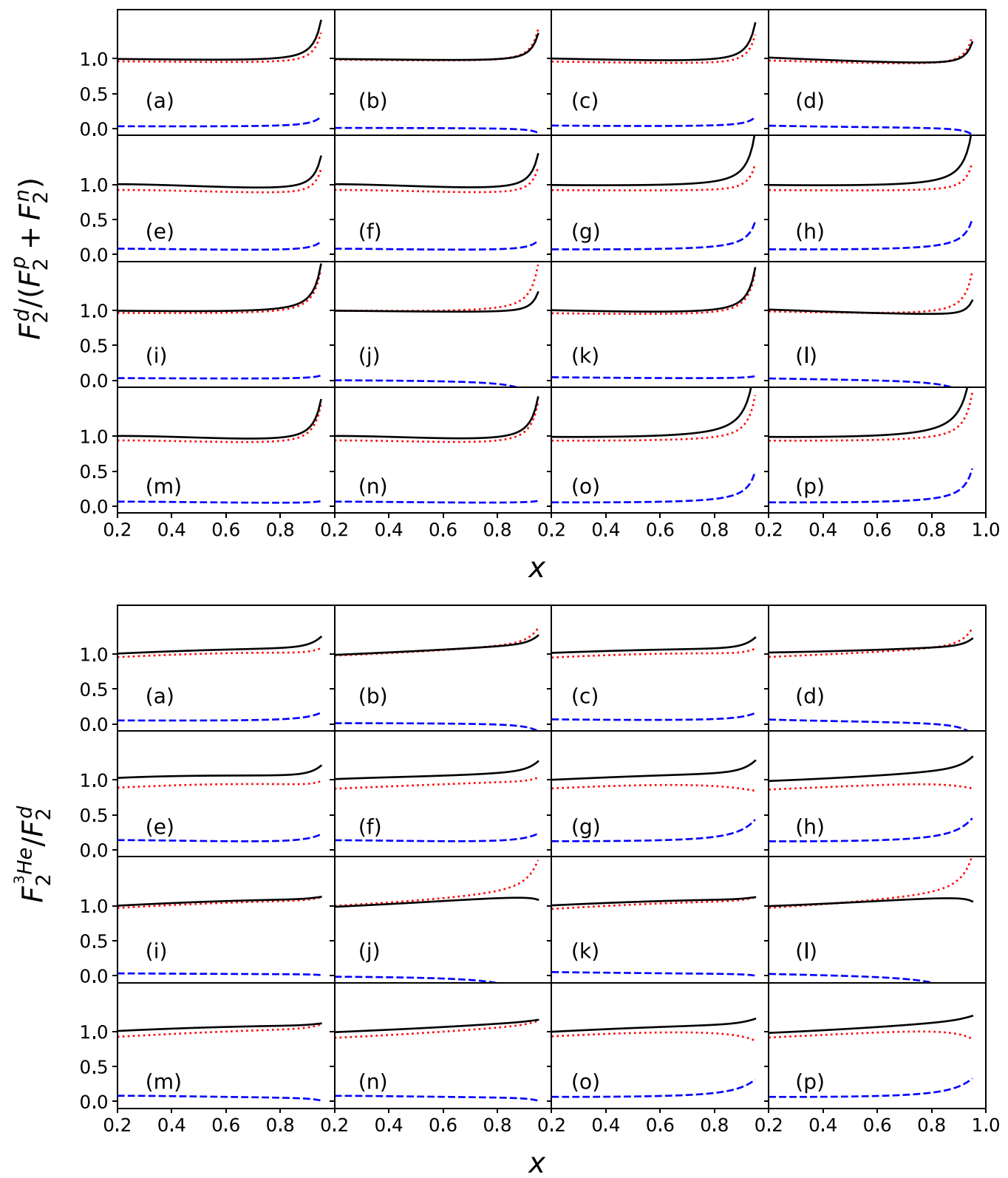

FIG. 19. Decomposition of EMC ratios (Top: deuterium, Bottom: helium-3) for MF (red finely dotted lines) and SRC (blue dashed lines) nucleons, compared to the full (black solid lines) convolution. See section on radio decomposition for details on calculations. In all curves we see that MF dominates the ratio and the SRC contribution is only $\mathcal{O}(\sim 10 \%)$. Labels for subplots signify fit results using model assumptions described in $\chi^{2}$-minimization procedure.

the largest deviations between these models occurs at large virtuality, in the SRC-dominated region. The SF model has increased probability of low- $\alpha$ nucleons relative to the GCF-LC model. In both formalisms, a momentum cutoff was placed at $p=1 \mathrm{GeV} / c$ due to the expectation that assumptions would break down beyond this region. The hard cuts seen at large $-v$ are the result of this cutoff, which is expressed differently in the GCF-LC model and the SF model.

Figure 2 shows the distribution $\tilde{\rho}(\alpha) / \alpha$ of the lightcone fraction for all nucleons (integrated over $v$ ), for deuterium and both protons and neutrons in helium-3. While the GCF-LC formalism produces lightcone fraction distributions that are symmetric around $\alpha=1$, this symmetry is not manifest in the SF formalism, resulting in the small momentum sum-rule violation mentioned in the main text.

\section{APPENDIX C: QUALITY OF MODEL EXTRACTION}

Each minimization trial is shown individually with the data used here with the resulting reduced $\chi^{2}$ in Fig. 12, and see Fig. 13 for equivalent Fig. 4 but using the GCF-LC approach.

\section{APPENDIX D: LOW $Q^{2}$, HIGH- $x_{B}$ SENSITIVITY}

In Eq. (1) of the main text and here, the Bjorken-limit was already invoked $\left(Q^{2}, q \rightarrow \infty, x\right.$ fixed). The full convolution 


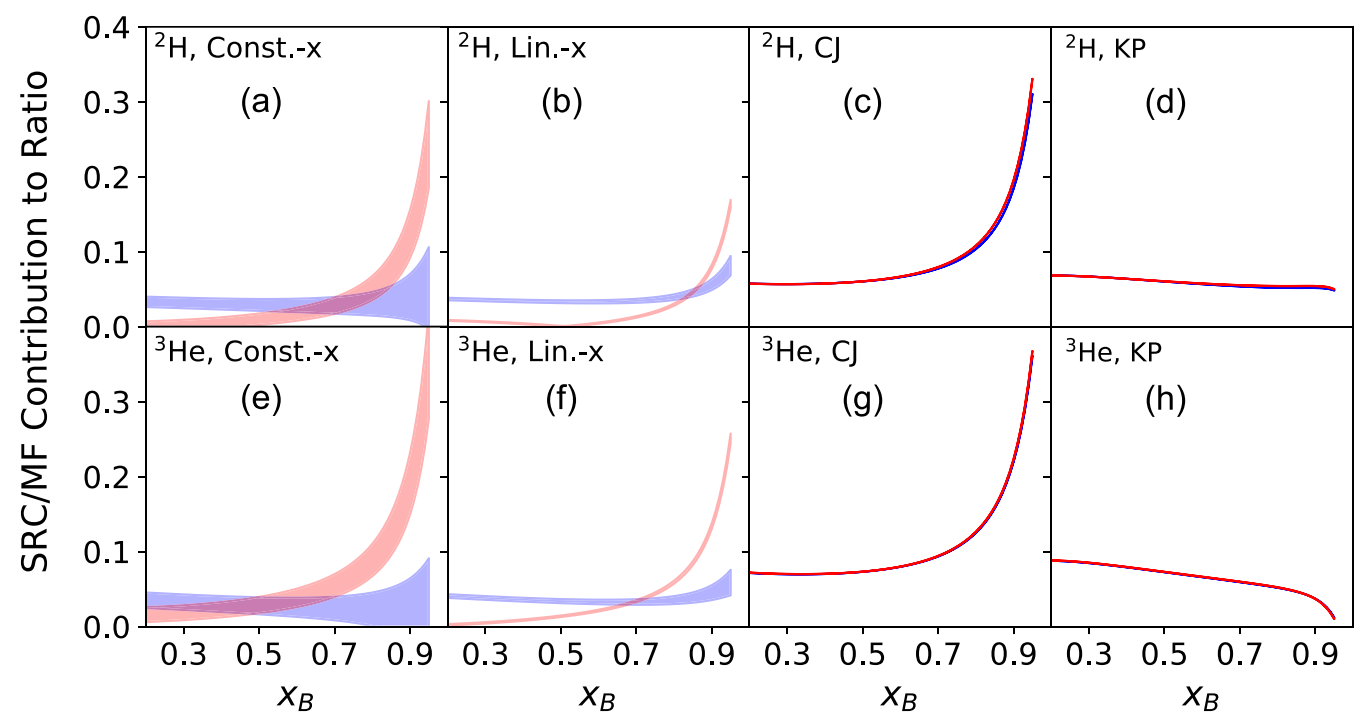

FIG. 20. Equivalent figure as Fig. 6 in the main text, but for the lightcone convolution approximation. Ratio of SRC contribution to MF contribution of the structure function. [Top row: (a)-(d)] Ratio on $F_{2}^{d}$. [Bottom row: (e)-(h)] Ratio on $F_{2}^{3} H e$. In each row, results are shown for different off-shell functions used, in order from left to right: $f_{\text {const }}^{\text {off }}[(\mathrm{a}),(\mathrm{e})], f_{\operatorname{lin} x}^{\text {off }}[(\mathrm{b}),(\mathrm{f})], f_{C J}^{\text {off }}[(\mathrm{c}),(\mathrm{g})], f_{K P}^{\text {off }}[(\mathrm{d}),(\mathrm{h})] .1 \sigma$ confidence bands are displayed for each trial. Curves are shown with $Q^{2}=5 \mathrm{GeV}^{2}$.

formula, as defined in [57] Eqs. (17) and (37), may be utilized to investigate finite- $Q^{2}$ effects. In order to study the size of these effects, we performed the convolution integral twice, once using the full formula including finite- $Q^{2}$ effects following Ref. [57], and once using the approximation of Eq. (1) with $\tilde{x}=\frac{x_{B}}{\alpha} \frac{A m_{N}}{m_{A}}$. We did this for both the spectral function and the lightcone convolution approximations. Although the data used in this analysis is at low-to-moderate $Q^{2}$, we find that our calculations are relatively insensitive to finite- $Q^{2}$ effects $(\sim 1-2 \%)$, especially considering that we utilize structure function ratios, which suppress these effects (see Fig. 14).

Another way to estimate the effects of finite $Q^{2}$ is the definition of $\tilde{x}$ used in the convolution. Defining $q=$ $(v, 0,0,-|\mathbf{q}|)$, we may express the momenta of the virtual photon and the struck nucleon in the frame:

$$
\begin{aligned}
& q=\left(q_{-}=\left(q_{0}-q_{3}\right), q_{+}=\left(q_{0}+q_{3}\right), \mathbf{q}_{\mathbf{t}}=0\right) \\
& p=\left(p_{-}=\left(p_{0}-p_{3}\right) / m_{N}, p_{+}=\left(p_{0}+p_{3}\right) / m_{N}, \mathbf{p}_{\mathbf{t}}\right),
\end{aligned}
$$

where in this frame, $q_{0}=v, q_{3}=|\mathbf{q}|$. Defining $\tilde{x}$ :

$$
\begin{aligned}
\tilde{x} & =\frac{Q^{2}}{2(q \cdot p)}=\frac{Q^{2}}{2\left(\left[q_{+} \cdot p_{-}\right] / 2+\left[q_{-} \cdot p_{+}\right] / 2-\mathbf{q}_{\mathbf{t}} \cdot \mathbf{p}_{\mathbf{t}}\right)} \\
& =\frac{Q^{2}}{\left[q_{+} \cdot p_{-}\right]+\left[q_{-} \cdot p_{+}\right]} .
\end{aligned}
$$

In the Bjorken frame, $\frac{q_{+}}{q_{-}} \rightarrow 0$ such that $\tilde{x} \rightarrow Q^{2} /\left(q_{-}\right.$. $\left.p_{+}\right)$. With fixed $x_{B}=Q^{2} /\left(2 m_{N} v\right), x_{B} \rightarrow Q^{2} / m_{N} q_{-}$:

$$
\tilde{x}=\frac{Q^{2}}{q_{-} \cdot p_{+}}=\frac{x_{B}}{p_{+}} m_{N}=\frac{x_{B}}{\alpha} \frac{A m_{N}}{m_{A}},
$$

where $\alpha=A p_{+} / m_{A}$. With this, we have arrived at the expression used in Eq. (1) in the main text. As the data considered in our fit is at finite- $Q^{2}$, we may also directly use Eq. (D2) for $\tilde{x}$. We repeated our minimization procedures and no significant difference was seen in the ratios $F_{2}^{d} /\left(F_{2}^{p}+F_{2}^{n}\right), F_{2}^{3} H e / F_{2}^{d}$ (see Fig. 15).

\section{APPENDIX E: TAGGED KINEMATICS}

When considering deuterium, in PWIA, we can write $\tilde{x}=$ $Q^{2} /(2 q \cdot p)$ using the kinematics of the spectator nucleon to ensure that as $\tilde{x} \rightarrow 1, \tilde{W} \rightarrow m_{N}^{2}$. Given that the initial deuterium nucleus is at rest $\left(p_{d}=\left(m_{d}, \mathbf{0}\right)\right)$ and the spectator nucleon is on-shell after the interaction:

$$
\tilde{x}_{\text {tagged }}=\frac{Q^{2}}{2\left[v\left(m_{d}-E_{r}\right)+\mathbf{p}_{r} \cdot \mathbf{q}\right]},
$$

where $\mathbf{p}_{r}=-\mathbf{p}$ and $E_{r}=\sqrt{m_{N}^{2}+|\mathbf{p}|^{2}}$. For the case of ${ }^{3} \mathrm{He}$, there are two-body breakup (2bbu) and three-body breakup (3bbu) channels that the resulting $A-1$ system can exist in. Defining a similar definition of Eq. (E1), we can then look at the impact of this on the ratios $F_{2}^{d} /\left(F_{2}^{p}+F_{2}^{n}\right)$ and $F_{2}^{3} H e / F_{2}^{d}$, see Fig. 16. In deuterium, the effect is on the order of $\sim 2 \%$ at $x_{B} \sim 0.7$. When calculating $F_{2}^{3} \mathrm{He}$, it is a slightly larger effect, however it is not much larger than $F_{2}^{d}$, which is why the ratio of $\left.\left(F_{2}^{3} \mathrm{He} / F_{2}^{d}\right)\right|_{\mathrm{Eq} .6} /\left.\left(F_{2}^{3} \mathrm{He} / F_{2}{ }^{d}\right)\right|_{\mathrm{Eq} .5}$ is small.

\section{APPENDIX F: UNIVERSAL FUNCTION PREDICTION}

We note that the UMF extracted by Ref. [7,21] is reproduced with the convolution framework used here for ${ }^{3} \mathrm{He}$, see Fig. 17.

\section{APPENDIX G: LOW-W RESONANCE CONTRIBUTIONS}

As the data considered in this analysis is at relatively low $W$, particularly at high $x_{B}$, we also investigated the contributions due to resonance effects in the underlying free structure functions $F_{2}^{p}$ and $F_{2}^{n}$. By using the Bosted-Christy param- 

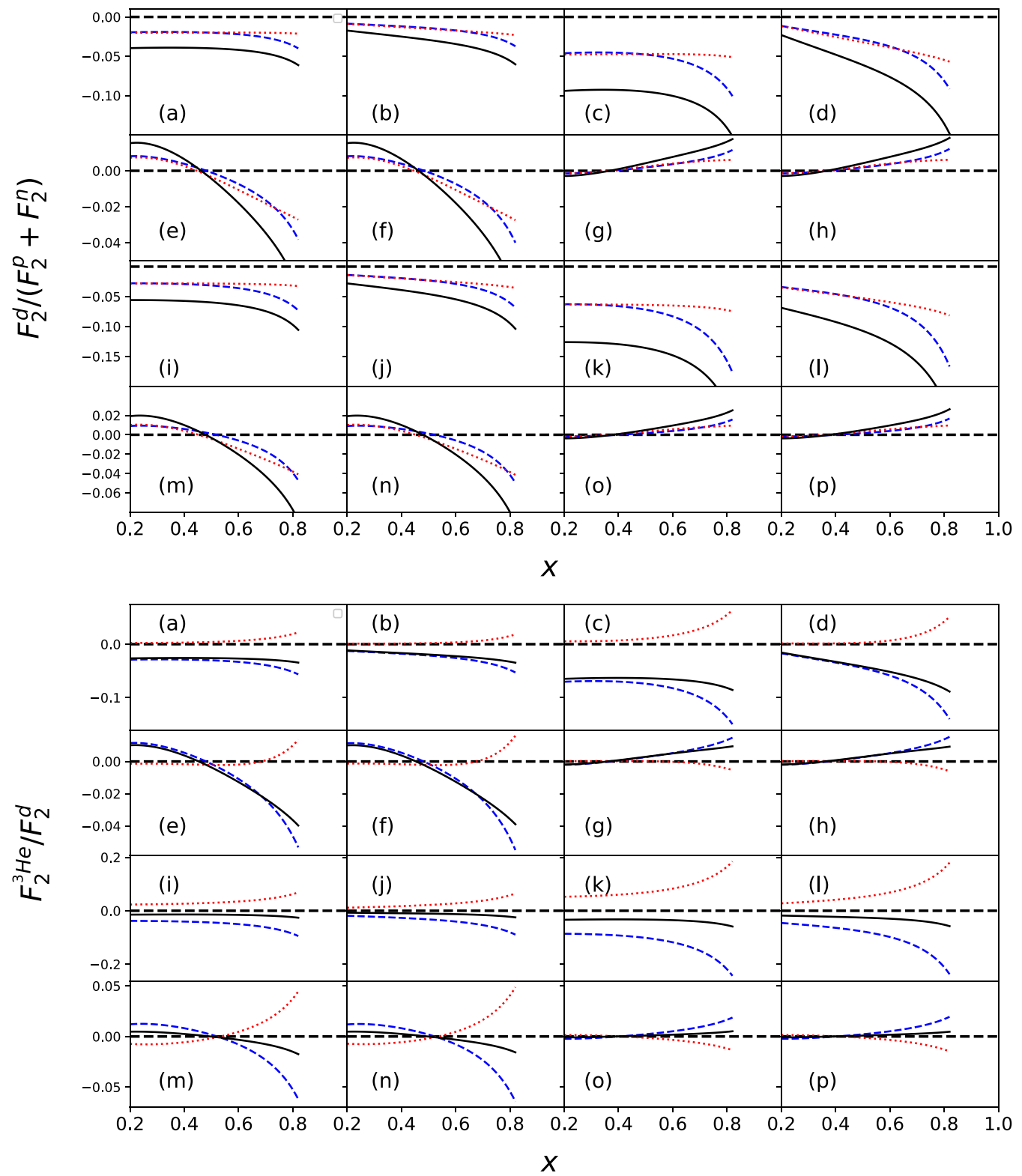

FIG. 21. Decomposition of off-shell contribution to ratio (Top: deuterium, Bottom: helium-3) for MF (red finely dotted lines) and SRC (blue dashed lines) nucleons, compared to the full (black solid lines) convolution. See section on off-shell decomposition for details on calculations. In most all curves we see that now SRC dominates the contribution at high $x_{B}$ and the MF contribution is small. Labels for subplots signify fit results using model assumptions described in the section on $\chi^{2}$-minimization procedure.

eterizations of $F_{2}^{p}$ and $F_{2}^{n}$ [50], resonance contributions to our convolution calculation of $F_{2}{ }^{d}$ and $F_{2}^{3} \mathrm{He}$ are considered. Furthermore, by utilizing these parameterizations, we allow for the separate $Q^{2}$ dependence of $F_{2}^{n}$ and $F_{2}^{p}$ in the ratio $F_{2}^{n}\left(\tilde{x}, Q^{2}\right) / F_{2}^{p}\left(\tilde{x}, Q^{2}\right)$ (in the main text it is assumed the $Q^{2}$ dependence in the ratio is negligible).

We repeated our entire analysis to infer the off-shell function with the Bosted-Christy parameterizations of $F_{2}^{p}\left(\tilde{x}, Q^{2}\right)$ and $F_{2}^{n}\left(\tilde{x}, Q^{2}\right)$ here. To investigate the sensitivity to resonance contributions, we only consider an off-shell function that is constant in $x, f_{\text {const }}^{\text {off }}$ and only consider the spectral function approximation to the convolution integral. We find a good description of the data, $\chi^{2} /$ dof $=25.5 / 31 \approx 0.82$ [compared to iteration (a) where we achieved a $\chi^{2} /$ dof $=19.8 / 31 \approx 0.63$ ].
Comparing to iteration (a), where we used the same off-shell functional form and spectral function approximation, we find consistent results for all of our findings in the main text, see Fig. 18. While the off-shell function is slightly reduced in magnitude, we find still that (1) the SRC/MF contribution to the structure function is small and (2) SRC nucleons play a large role in the off-shell contribution to the structure function.

\section{APPENDIX H: CONVOLUTION DECOMPOSITION}

\section{Ratio decomposition}

In calculating Eq. (A1), we would like to understand the contribution of low- and high-momentum nucle- 


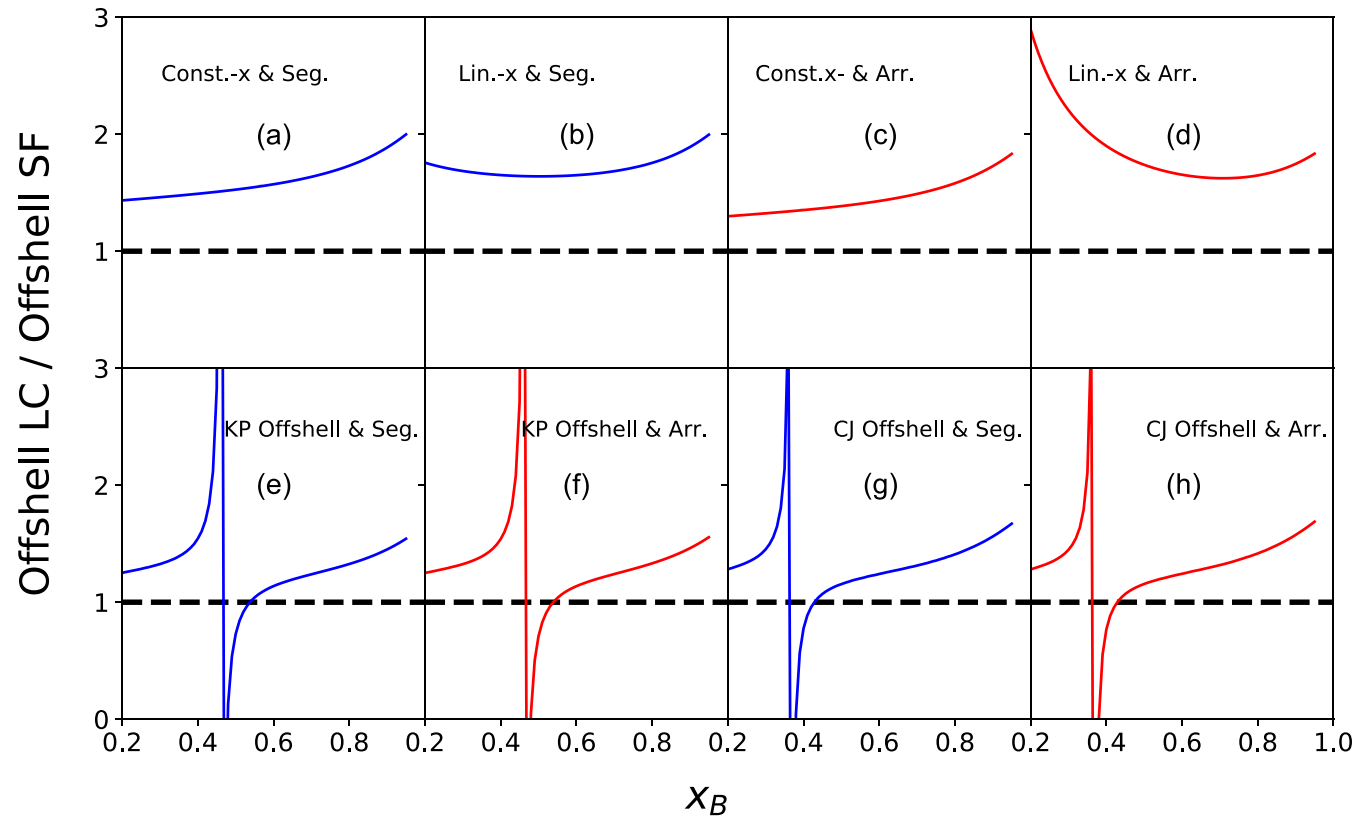

FIG. 22. Ratio of of-fshell contribution in Helium-3 to the structure function in the GCF-LC framework to SF framework. The ratio is always larger than one, signifying that the off-shell effect is larger in the GCF-LC framework than SF framework, as expected due to the momentum sum rule violation in the SF framework. All blue lines use $F_{2}^{n} /\left.F_{2}^{p}\right|_{\text {Seg. }}$ [(a),(b),(e),(g)], whereas red lines use $F_{2}^{n} /\left.F_{2}^{p}\right|_{\text {Arr. }}$ $[(\mathrm{c}),(\mathrm{d}),(\mathrm{f}),(\mathrm{h})]$. The off-shell functional form utilized is written in each subfigure.

ons to the full nuclear structure function. In the GCFLC formalism, $\tilde{\rho}(\alpha, v)$ is already factorized into meanfield/low-momentum (MF) and short-range-correlation/high- momentum (SRC) contributions, see Ref. [38]. In the SF approximation, we simply choose integral bounds of the spectral function for the momentum range of interest (we

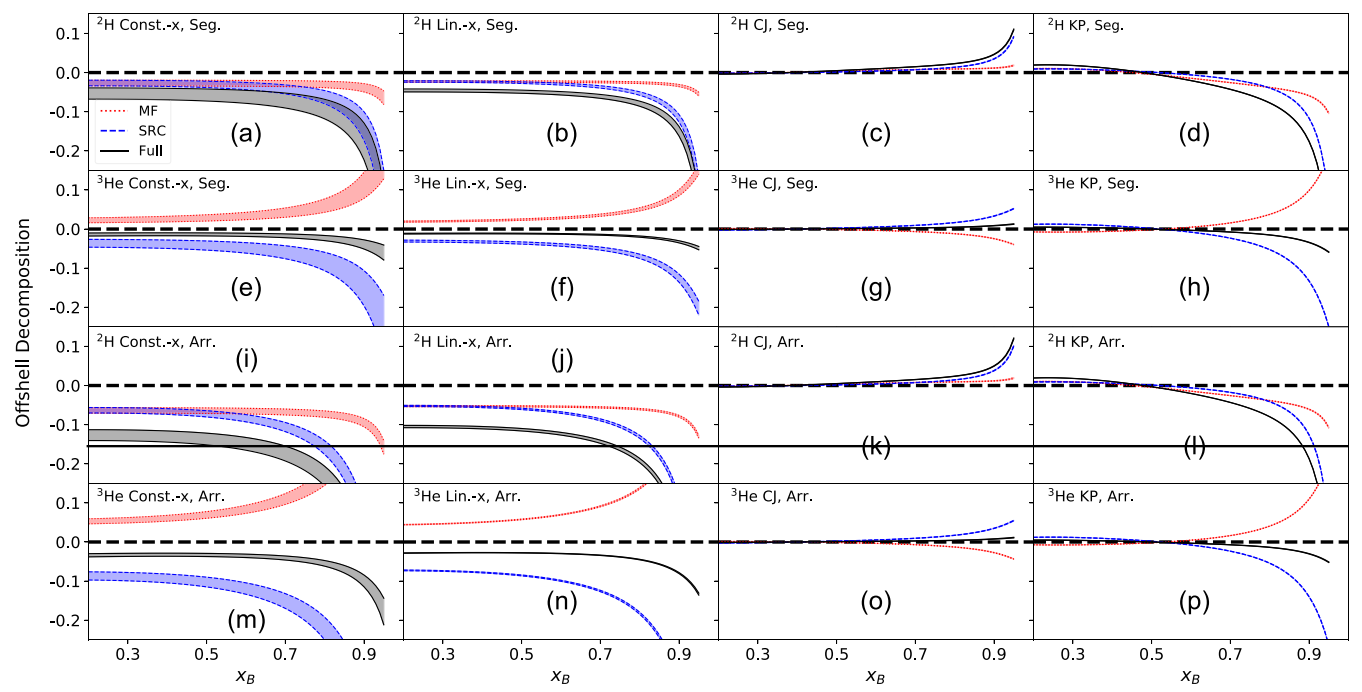

FIG. 23. Equivalent figure as Fig. 7 in the main text, but for the lightcone convolution approximation. Decomposition of $F_{2}^{A}$ (off-shell) for various model assumptions. [Top row, (a)-(d)] Decomposition for $F_{2}^{d} /\left(F_{2}^{p}+F_{2}^{n}\right)$ with Segarra et al. $F_{2}^{n} / F_{2}^{p}$. [2nd row, (e)-(h)] Decomposition for $F_{2}^{3} \mathrm{He} / F_{2}^{d}$ with Segarra et al. $F_{2}^{n} / F_{2}^{p}$. [3rd row, (i)-(1)] Decomposition for $F_{2}^{d} /\left(F_{2}^{p}+F_{2}^{n}\right)$ with Arrington $F_{2}^{n} / F_{2}^{p}$. [Bottom row, (m)-(p)] Decomposition for $F_{2}^{3} \mathrm{He} / F_{2}{ }^{d}$ with Arrington $F_{2}^{n} / F_{2}^{p}$. In each row, decompositions are shown for different off-shell functions used, in order from left to right: $f_{\text {const }}^{\text {off }}[(\mathrm{a}),(\mathrm{e}),(\mathrm{i}),(\mathrm{m})], f_{\operatorname{lin} x}^{\text {off }}[(\mathrm{b}),(\mathrm{f}),(\mathrm{j}),(\mathrm{n})], f_{C J}^{\text {off }}[(\mathrm{c}),(\mathrm{g}),(\mathrm{k}),(\mathrm{o})], f_{K P}^{\text {off }}[(\mathrm{d}),(\mathrm{h}),(\mathrm{l}),(\mathrm{p})]$. Solid black lines represent the full off-shell contribution. Dashed blue lines are the contribution due to SRC nucleons ( $>240 \mathrm{MeV} / \mathrm{c}$ in the SF assumption). Similarly, dotted red lines are the contribution due to $\mathrm{MF}$ nucleons $(<240 \mathrm{MeV} / \mathrm{c}) .1 \sigma$ confidence bands are displayed for each trial. Curves are shown at $Q^{2}=5 \mathrm{GeV}^{2}$. 
chose $240 \mathrm{MeV} / \mathrm{c})$ :

$$
\begin{aligned}
\tilde{\rho}_{N, S F}^{S R C, A}(\alpha, v)= & \int_{|\mathbf{p}|=240 \mathrm{MeV} / \mathrm{c}}^{\infty} d E d^{3} \mathbf{p} S_{N}^{A}(E, p) \\
& \times \frac{E+p_{z}}{E} \cdot \frac{m_{A}}{m_{N} A} \delta\left(\frac{m_{N} \alpha}{m_{A}}-\frac{p^{+}}{P^{+}}\right) \\
& \times \delta\left(v-\frac{E^{2}-|\mathbf{p}|^{2}-m_{N}^{2}}{m_{N}^{2}}\right),
\end{aligned}
$$

and similarly, a $\tilde{\rho}_{N, S F}^{M F, A}(\alpha, v)$ is defined by integrating from $0-240 \mathrm{MeV} / \mathrm{c}$. Substituting in $\tilde{\rho}_{N}^{S R C, A}(\alpha, v)$ into Eq. (A1), we can calculate $F_{2}^{S R C, A}$, the contribution to $F_{2}^{A}$ by SRC nucleons. We note that $F_{2}^{M F, A}+F_{2}^{S R C, A}=F_{2}^{A}$. Figure 19 shows the individual contributions to $F_{2}^{A}$ by $\mathrm{MF}$ and SRC nucleons (in the main text, the ratio of SRC/MF was taken, and see Fig. 20 for equivalent Fig. 6 but using the GCF-LC approach).

\section{Off-shell decomposition}

We would also like to understand the role of high momentum nucleons in the of-fshell effect $f^{\text {off }}(\tilde{x})$ and how that contributes to the overall structure function. We can estimate the of-fshell contribution to the structure function by taking $F_{2}^{A}$ (off-shell) $=F_{2}^{A}$ (full) $-F_{2}^{A}$ (no-offshell), where we calculate $F_{2}^{A}$ (full) by usual means of Eq. (A1) and $F_{2}^{A}$ (no-offshell) is calculated by setting $f^{\text {off }}(\tilde{x})=0$ in Eq. (A1). We then can look again at the contributions due to $\mathrm{MF}$ and SRC nucleons to $F_{2}^{A}$ (off-shell). Figure 21 shows the individual contributions to $F_{2}^{A}$ (off-shell) by MF and SRC nucleons (in the main text, only a select few curves were shown). As was noted in the main text, in the SF framework, there is a slight momentum sum rule violation in helium-3, which induces an artificial off-shell effect. In Fig. 22, we look at the ratio of the off-shell effect in helium-3 using the GCF-LC approach and the SF approach. The GCF-LC framework requires a larger off-shell effect, as expected due to manifestly satisfying the momentum sum rule. See Fig. 23 for equivalent Fig. 7 but for the GCF-LC approach.
[1] R. G. Arnold, P. E. Bosted, C. C. Chang, J. Gomez, A. T. Katramatou, G. G. Petratos, A. A. Rahbar, S. E. Rock, A. F. Sill, Z. M. Szalata et al., Measurements of the $a$ Dependence of Deep-Inelastic Electron Scattering from Nuclei, Phys. Rev. Lett. 52, 727 (1984).

[2] J. J. Aubert et al., The ratio of the nucleon structure functions $F_{2}^{N}$ for iron and deuterium, Phys. Lett. B 123, 275 (1983).

[3] J. Ashman et al., Measurement of the ratios of deep inelastic muon-nucleus cross sections on various nuclei compared to deuterium, Phys. Lett. B 202, 603 (1988).

[4] J. Gomez, R. G. Arnold, P. E. Bosted, C. C. Chang, A. T. Katramatou, G. G. Petratos, A. A. Rahbar, S. E. Rock, A. F. Sill, Z. M. Szalata et al., Measurement of the $A$ dependence of deep-inelastic electron scattering, Phys. Rev. D 49, 4348 (1994).

[5] M. Arneodo et al., Measurements of the nucleon structure function in the range $0.002<x<0.17$ and $0.2<q^{2}<8$ gev $^{2}$ in deuterium, carbon and calcium, Nucl. Phys. B 333, 1 (1990).

[6] J. Seely et al., New Measurements of the European Muon Collaboration Effect in Very Light Nuclei, Phys. Rev. Lett. 103, 202301 (2009).

[7] B. Schmookler et al. (The CLAS Collaboration), Modified structure of protons and neutrons in correlated pairs, Nature 566, 354 (2019).

[8] L. Frankfurt and M. Strikman, Hard nuclear processes and microscopic nuclear structure, Phys. Rep. 160, 235 (1988).

[9] M. M. Sargsian et al., Hadrons in the nuclear medium, J. Phys. G 29, R1 (2003).

[10] P. R. Norton, The EMC effect, Rep. Prog. Phys. 66, 1253 (2003).

[11] O. Hen, G. A. Miller, E. Piasetzky, and L. B. Weinstein, Nucleon-nucleon correlations, short-lived excitations, and the quarks within, Rev. Mod. Phys. 89, 045002 (2017).

[12] I. C. Cloet, W. Bentz, and A. W. Thomas, Isovector EMC Effect Explains the NuTeV Anomaly, Phys. Rev. Lett. 102, 252301 (2009).
[13] I. C. Cloët, W. Bentz, and A. W. Thomas, Parity-Violating Deep Inelastic Scattering and the Flavor Dependence of the EMC Effect, Phys. Rev. Lett. 109, 182301 (2012).

[14] S. Malace, D. Gaskell, D. W. Higinbotham, and I. Cloet, The challenge of the EMC effect: Existing data and future directions, Int. J. Mod. Phys. E 23, 1430013 (2014).

[15] W. Melnitchouk, A. W. Schreiber, and A. W. Thomas, Deep inelastic scattering from off-shell nucleons, Phys. Rev. D 49 , 1183 (1994).

[16] O. Benhar, S. Fantoni, and G. I. Lykasov, Nuclear structure functions and off-shell corrections, Phys. Lett. B 502, 69 (2001).

[17] D. M. Alde, H. W. Baer, T. A. Carey, G. T. Garvey, A. Klein, C. Lee, M. J. Leitch, J. W. Lillberg, P. L. McGaughey, C. S. Mishra et al., Nuclear Dependence of Dimuon Production at $800-\mathrm{GeV}$. FNAL-772 Experiment, Phys. Rev. Lett. 64, 2479 (1990).

[18] L. B. Weinstein, E. Piasetzky, D. W. Higinbotham, J. Gomez, O. Hen, and R. Shneor, Short Range Correlations and the EMC Effect, Phys. Rev. Lett. 106, 052301 (2011).

[19] O. Hen, E. Piasetzky, and L. B. Weinstein, New data strengthen the connection between short range correlations and the emc effect, Phys. Rev. C 85, 047301 (2012).

[20] O. Hen, D. W. Higinbotham, G. A. Miller, E. Piasetzky, and L. B. Weinstein, The EMC effect and high momentum nucleons in nuclei, Int. J. Mod. Phys. E 22, 1330017 (2013).

[21] E. P. Segarra, A. Schmidt, T. Kutz, D. W. Higinbotham, E. Piasetzky, M. Strikman, L. B. Weinstein, and O. Hen, Neutron Valence Structure from Nuclear Deep Inelastic Scattering, Phys. Rev. Lett. 124, 092002 (2020).

[22] S. A. Kulagin and R. Petti, Global study of nuclear structure functions, Nucl. Phys. A 765, 126 (2006).

[23] A. J. Tropiano, J. J. Ethier, W. Melnitchouk, and N. Sato, Deepinelastic and quasielastic electron scattering from $a=3$ nuclei, Phys. Rev. C 99, 035201 (2019).

[24] G. G. Petratos et al., MeAsurement of the $F_{2}^{n} / F_{2}^{p}, d / u$ RAtios and $A=3$ EMC Effect in Deep Inelastic Electron Scattering Off 
the Tritium and Helium MirrOr Nuclei, Jefferson Lab PAC36 Proposal (2010).

[25] O. Hen et al., "In medium proton structure functions, src, and the EMC effect," Jefferson Lab PAC Proposal (2015).

[26] O. Hen, L.B. Weinstein, S.A. Wood, and S. Gilad, "In Medium Nucleon Structure Functions, SRC, and the EMC effect, Jefferson Lab experiment E12-11-107," (2011).

[27] L. L. Frankfurt and M. I. Strikman, Point-like configurations in hadrons and nuclei and deep inelastic reactions with leptons: EMC and EMC like effects, Nucl. Phys. B 250, 143 (1985).

[28] L. L. Frankfurt and M. I. Strikman, On the normalization of nucleus spectral function and the EMC effect, Phys. Lett. B 183, 254 (1987).

[29] S. V. Akulinichev, S. Shlomo, S. A. Kulagin, and G. M. Vagradov, Lepton-Nucleus Deep-Inelastic Scattering, Phys. Rev. Lett. 55, 2239 (1985).

[30] G. V. Dunne and A. W. Thomas, The effect of conventional nuclear binding on nuclear structure functions, Nucl. Phys. A 455, 701 (1985).

[31] G. A. Miller, Confinement in Nuclei and the Expanding Proton, Phys. Rev. Lett. 123, 232003 (2019).

[32] H. Jung and G. A. Miller, Pionic contributions to deep inelastic nuclear structure functions, Phys. Rev. C 41, 659 (1990).

[33] H. Jung and G. A. Miller, Nucleonic contribution to leptonnucleus deep inelastic scattering, Phys. Lett. B 200, 351 (1988).

[34] C. Ciofi degli Atti and L. P. Kaptari, Calculations of the Exclusive Processes ${ }^{2} \mathrm{H}\left(e, e^{\prime} p\right) n,{ }^{3} \mathrm{He}\left(e, e^{\prime} p\right){ }^{2} \mathrm{H}$ and ${ }^{3} \mathrm{He}\left(e, e^{\prime} p\right)(p n)$ within a generalized eikonal approximation, Phys. Rev. C 71, 024005 (2005).

[35] C. Ciofi degli Atti and S. Simula, Realistic model of the nucleon spectral function in few and many nucleon systems, Phys. Rev. C 53, 1689 (1996).

[36] C. Ciofi degli Atti, L. L. Frankfurt, L. P. Kaptari, and M. I. Strikman, Dependence of the wave function of a bound nucleon on its momentum and the EMC effect, Phys. Rev. C 76, 055206 (2007).

[37] R. Weiss, I. Korover, E. Piasetzky, O. Hen, and N. Barnea, Energy and momentum dependence of nuclear short-range correlations - Spectral function, exclusive scattering experiments and the contact formalism, Phys. Lett. B 791, 242 (2019).

[38] J. R. Pybus, I. Korover, R. Weiss, A. Schmidt, N. Barnea, D. W. Higinbotham, E. Piasetzky, M. Strikman, L. B. Weinstein, and $\mathrm{O}$. Hen, Generalized contact formalism analysis of the ${ }^{4} \mathrm{He}\left(e, e^{\prime} p N\right)$ reaction, Phys. Lett. B 805, 135429 (2020).

[39] A. Schmidt et al. (The CLAS Collaboration), Probing the core of the strong nuclear interaction, Nature 578, 540 (2020).

[40] R. Weiss, R. Cruz-Torres, N. Barnea, E. Piasetzky, and O. Hen, The nuclear contacts and short range correlations in nuclei, Phys. Lett. B 780, 211 (2018).

[41] R. Cruz-Torres, D. Lonardoni, R. Weiss, M. Piarulli, N. Barnea, D. W. Higinbotham, E. Piasetzky, A. Schmidt, L. B. Weinstein,
R. B. Wiringa, and O. Hen, Many-Body factorization and position-momentum equivalence of nuclear short-range correlations, Nat. Phys. 17, 306 (2020).

[42] K. Egiyan et al. (CLAS Collaboration), Measurement of 2and 3-Nucleon Short Range Correlation Probabilities in Nuclei, Phys. Rev. Lett. 96, 082501 (2006).

[43] O. Benhar, A. Fabrocini, and S. Fantoni, Nuclear-matter green functions in correlated-basis theory, Nucl. Phys. A 550, 201 (1992).

[44] O. Benhar, A. Fabrocini, and S. Fantoni, Occupation probabilities and hole-state strengths in nuclear matter, Phys. Rev. C 41, R24 (1990).

[45] I. Sick, S. Fantoni, A. Fabrocini, and O. Benhar, Spectral function of medium-heavy nuclei and electron scattering, Phys. Lett. B 323, 267 (1994).

[46] A. Accardi, L. T. Brady, W. Melnitchouk, J. F. Owens, and N. Sato, Constraints on large- $x$ parton distributions from new weak boson production and deep-inelastic scattering data, Phys. Rev. D 93, 114017 (2016).

[47] J. Arrington, J. G. Rubin, and W. Melnitchouk, How Well Do We Know The Neutron Structure Function? Phys. Rev. Lett. 108, 252001 (2012).

[48] A. Airapetian, N. Akopov et al. (The HERMES Collaboration), Inclusive measurements of inelastic electron and positron scattering from unpolarized hydrogen and deuterium targets, J. High Energ. Phys. 05 (2011) 126.

[49] K. A. Griffioen, J. Arrington, M. E. Christy, R. Ent, N. Kalantarians, C. E. Keppel, S. E. Kuhn, W. Melnitchouk, G. Niculescu, I. Niculescu et al., Measurement of the EMC effect in the deuteron, Phys. Rev. C 92, 015211 (2015).

[50] P. E. Bosted and M. E. Christy, Empirical fit to inelastic electron-deuteron and electron-neutron resonance region transverse cross sections, Phys. Rev. C 77, 065206 (2008).

[51] S. Veerasamy and W. N. Polyzou, Momentum-space argonne v18 interaction, Phys. Rev. C 84, 034003 (2011).

[52] L. L. Frankfurt and M. I. Strikman, On the problem of extracting the neutron structure function from ed scattering, Phys. Lett. B 76, 333 (1978).

[53] X. G. Wang, A. W. Thomas, and W. Melnitchouk, Do ShortRange Correlations Cause the Nuclear EMC Effect in the Deuteron? Phys. Rev. Lett. 125, 262002 (2020).

[54] W. Melnitchouk, M. Sargsian, and M. Strikman, Probing the origin of the EMC effect via tagged structure functions of the deuteron, Z. Phys. A 359, 99 (1997).

[55] O. Hen et al., Momentum sharing in imbalanced Fermi systems, Science 346, 614 (2014).

[56] M. Duer et al. ( CLAS Collaboration), Probing high-momentum protons and neutrons in neutron-rich nuclei, Nature 560, 617 (2018).

[57] M. M. Sargsian, S. Simula, and M. I. Strikman, Neutron structure function and inclusive deep inelastic scattering from ${ }^{3} \mathrm{H}$ and ${ }^{3} \mathrm{He}$ at large Bjorken $x$, Phys. Rev. C 66, 024001 (2002). 